\title{
Aqueous outflow imaging techniques and what they tell us about intraocular pressure regulation
}

\author{
Jed A. Lusthaus $\mathbb{1}^{1,2} \cdot$ Tasneem Z. Khatib ${ }^{3,4} \cdot$ Paul A. R. Meyer ${ }^{5,6} \cdot$ Peter McCluskey ${ }^{1,2} \cdot$ Keith R. Martin ${ }^{2,3,4,7,8,9}$
}

Received: 9 July 2020 / Revised: 19 July 2020 / Accepted: 7 August 2020 / Published online: 21 August 2020

(c) The Author(s), under exclusive licence to The Royal College of Ophthalmologists 2020

\begin{abstract}
Recent advances in the medical and surgical management of open-angle glaucoma have increased the number of treatment options available. Several new intraocular pressure (IOP)-lowering treatments target the conventional aqueous outflow (AO) system. However, success rates are variable and outcomes in individual patients are often difficult to predict. Variable treatment responses remain unexplained and highlight deficiencies in our current understanding of AO regulation and IOP homeostasis. Imaging is often relied upon to confirm diagnoses and monitor treatment responses in other ocular and systemic pathologies. As yet no suitable AO imaging tool has been developed to fulfil this role in glaucoma. A variety of imaging techniques have been used to study the AO tracts of humans and animals in ex vivo and in vivo eyes. In this review, results from novel imaging techniques that assess aqueous drainage through the episcleral venous system are considered and we argue these provide new insights into AO regulation. We suggest that the ability to objectively measure AO responses to interventions would be a significant clinical advance, and we have demonstrated that this can be achieved with direct visualisation of aqueous drainage. We predict that the evolution of AO imaging technology will continue to reveal critical components of AO and IOP regulation, and that personalised IOP-lowering treatment in glaucoma care may well become a reality in the near future.
\end{abstract}

Jed A. Lusthaus

jed.lusthaus@sydney.edu.au

1 Department of Ophthalmology, Sydney Eye Hospital, Sydney, NSW, Australia

2 Discipline of Ophthalmology, The University of Sydney, Sydney, NSW, Australia

3 John van Geest Centre for Brain Repair, University of Cambridge, Cambridge, UK

4 Eye Department, Cambridge University Hospitals NHS Foundation Trust, Cambridge, UK

5 Department of Engineering, University of Cambridge, Cambridge, UK

6 Department of Medicine, University of Cambridge, Cambridge, UK

7 Ophthalmology, Department of Surgery, University of Melbourne, Melbourne, VIC, Australia

8 Centre for Eye Research Australia, The Royal Victorian Eye and Ear Hospital, Melbourne, VIC, Australia

9 Wellcome Trust-MRC Cambridge Stem Cell Institute, University of Cambridge, Cambridge, UK

\section{Introduction}

\section{Intraocular pressure and aqueous outflow}

The spherical shape of the eye is maintained by the stretching of its elastic, collagenous envelope by its incompressible contents. The force returned by this corneo-scleral extension and the balance between aqueous secretion and drainage determine baseline intraocular pressure (IOP). However, higher-frequency fluctuations are superimposed over this baseline pressure, as a result of variations in the volume of the intraocular circulations during the cardiac cycle, and forces acting on the globe due to contraction of extraocular muscles.

Aqueous outflow (AO) is considered to be the major determinant of baseline intraocular pressure, and increased outflow impedance is an important risk factor for glaucoma. The majority of aqueous leaves the globe by the "conventional" pathway, traversing the trabeculum to enter the circumferential canal of Schlemm, from where it is delivered by drainage channels into episcleral veins on the ocular surface. Those veins in which aqueous maintains a visible, discrete column are called aqueous veins. The episcleral veins disgorge into the superior and inferior ophthalmic 
veins, which traverse the orbit, delivering their contents into the cavernous sinus.

Imaging of $\mathrm{AO}$ has largely concentrated on its emergence from the globe; however, this review will also consider techniques that illustrate its passage through the episcleral circulation.

\section{Imaging of aqueous outflow}

Renewed interest in AO imaging has been generated by the introduction of minimally invasive glaucoma surgery (MIGS). A number of MIGS devices improve conventional AO by reducing outflow resistance, but IOP responses are unpredictable. This raises questions about the functionality of the conventional aqueous outflow system in glaucoma patients and what causes IOP dysregulation.

Elevated IOP is widely accepted as a major risk factor for primary open-angle glaucoma (POAG). IOP reduction has been successfully achieved by means of topical eye drops, laser and surgery. Interventions have emerged that augment conventional or uveoscleral aqueous outflow, or reduce aqueous production. As the size of the glaucoma population increases globally, there is greater demand for effective and consistent treatment responses so that outcomes and efficiency of care can be optimised. Novel treatment solutions are promising, but a greater understanding of functional $\mathrm{AO}$ is required to optimise treatment selection and timing. The mechanisms responsible for IOP dysregulation are elusive, although multiple findings from imaging studies support the presence of feedback control of aqueous drainage within the conventional AO system [1-6].

For the purpose of this review, we concentrated on the imaging of conventional aqueous outflow. Uveoscleral (unconventional) outflow represents a small proportion of total aqueous drainage [7] and cannot be accurately imaged [8]. AO imaging techniques can be classified in many ways, but are commonly described as structural or functional $[8,9]$. Structural imaging techniques identify anatomical features that may explain how aqueous passes through the conventional outflow system, whereas functional techniques study actual flow through the system. Studies include ex vivo and in vivo eyes of humans and animals. Available imaging techniques vary in invasiveness and in whether static or dynamic (real-time) assessments of AO are possible. Using a non-invasive imaging system to study AO in physiological conditions is desirable and may create opportunities to move towards more personalised glaucoma care. AO imaging techniques are summarised in Table 1.

\section{Methods}

Searches of PubMed, Medline, Google Scholar, Embase and Scopus were completed by 12th April 2020. Other sources were obtained from reference lists of selected studies, conference proceedings and generally available specialised websites. All relevant materials were included and not limited by date or original language of the publication.

\section{Background}

The first aqueous vein was reported in 1942 by Ascher, using slit lamp biomicroscopy [1]. Prior to this, multiple descriptions, most likely representing aqueous flow within episcleral vessels, date back to 1921. Gartner published the first aqueous vein photograph in 1944 [1], but detailed anatomical representations of $\mathrm{AO}$ channels have, until recently, only been possible in ex vivo eyes. In 1951, Ashton elegantly displayed the pathways of aqueous veins, derived from Schlemm's canal (SC), with the use of neoprene casts (Fig. 1) [10]. These images are often a prelude to discussions of AO. Now, with new technologies, AO imaging has been made possible in vivo and this enables functional assessment of flow. The goal of imaging this region of the eye is primarily to explain mechanisms of IOP control that may lead to new therapeutic targets or assist with monitoring responses to treatment. The following section summarises imaging techniques used to study the aqueous outflow system, and discusses how they may help to identify regulatory mechanisms.

\section{Structural aqueous outflow imaging}

It has been widely documented that aqueous is secreted by the ciliary body epithelium and drains through the trabecular meshwork (TM) into SC and the episcleral venous system (EVS) [9, 11]. Imaging the structural anatomy of the distal AO pathway, from SC into collector channels and episcleral veins, was initially made possible with the use of animal models and human ex vivo eyes.

\section{Microscopy}

Early use of imaging techniques on ex vivo primate, bovine and human eyes include light microscopy, scanning electron microscopy and transmission electron microscopy [12-16]. These techniques confirmed the drainage of aqueous and the anatomy of the AO drainage pathway (Fig. 2). The major anatomical contributors to $\mathrm{AO}$ include: the TM, the juxtacanalicular connective tissue (JCT), SC endothelium, collector channels and the episcleral venous system [17]. The inner wall of SC, comprised of the JCT and SC endothelium, is widely considered to be the predominant site of $\mathrm{AO}$ resistance [15-20]. Valve-like structures visualised after mechanical dilation of SC with viscoelastic $[14,21]$ are thought to aid pulsatile aqueous drainage through the outflow system [2]. These anatomical findings form the 


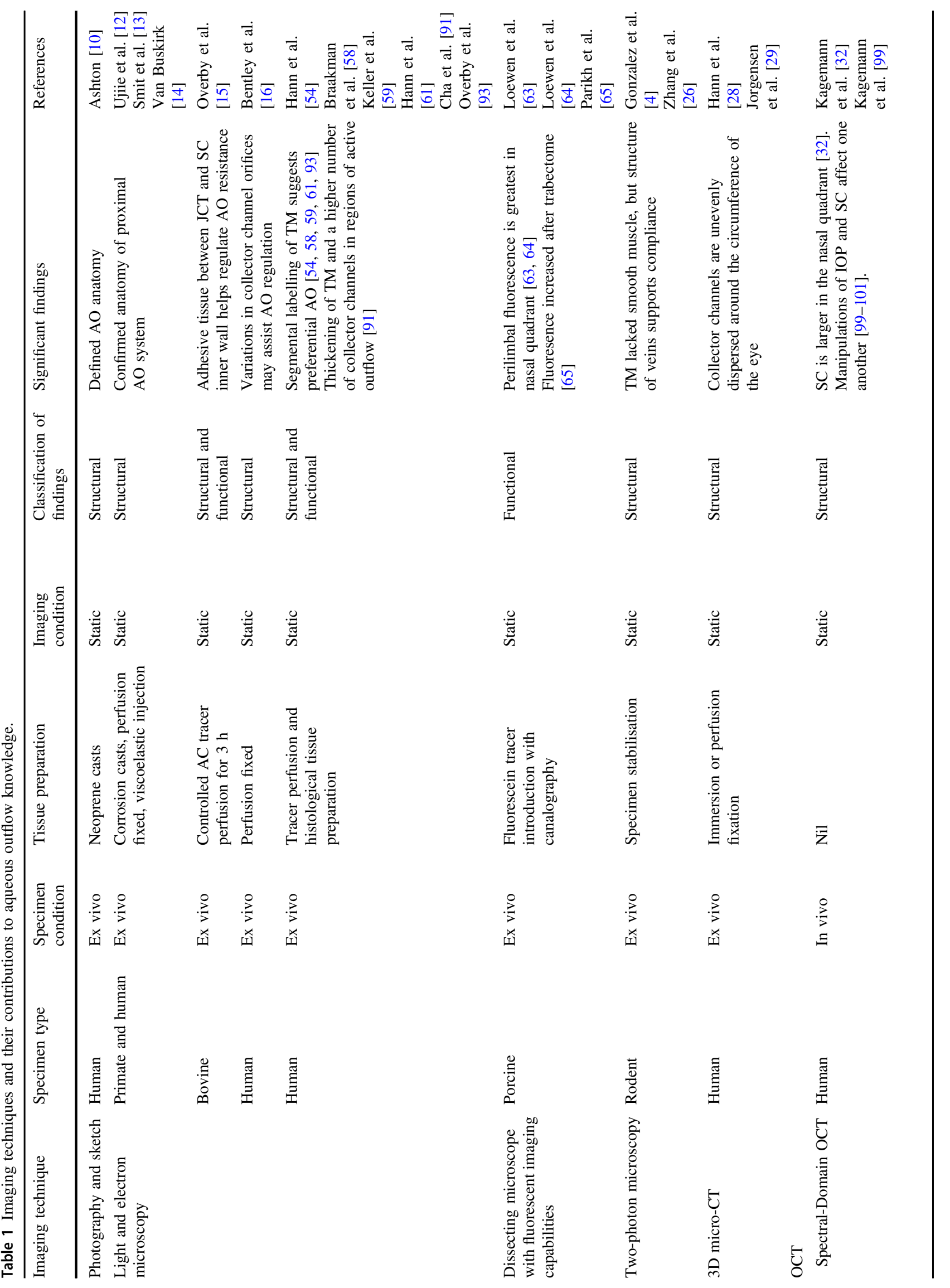




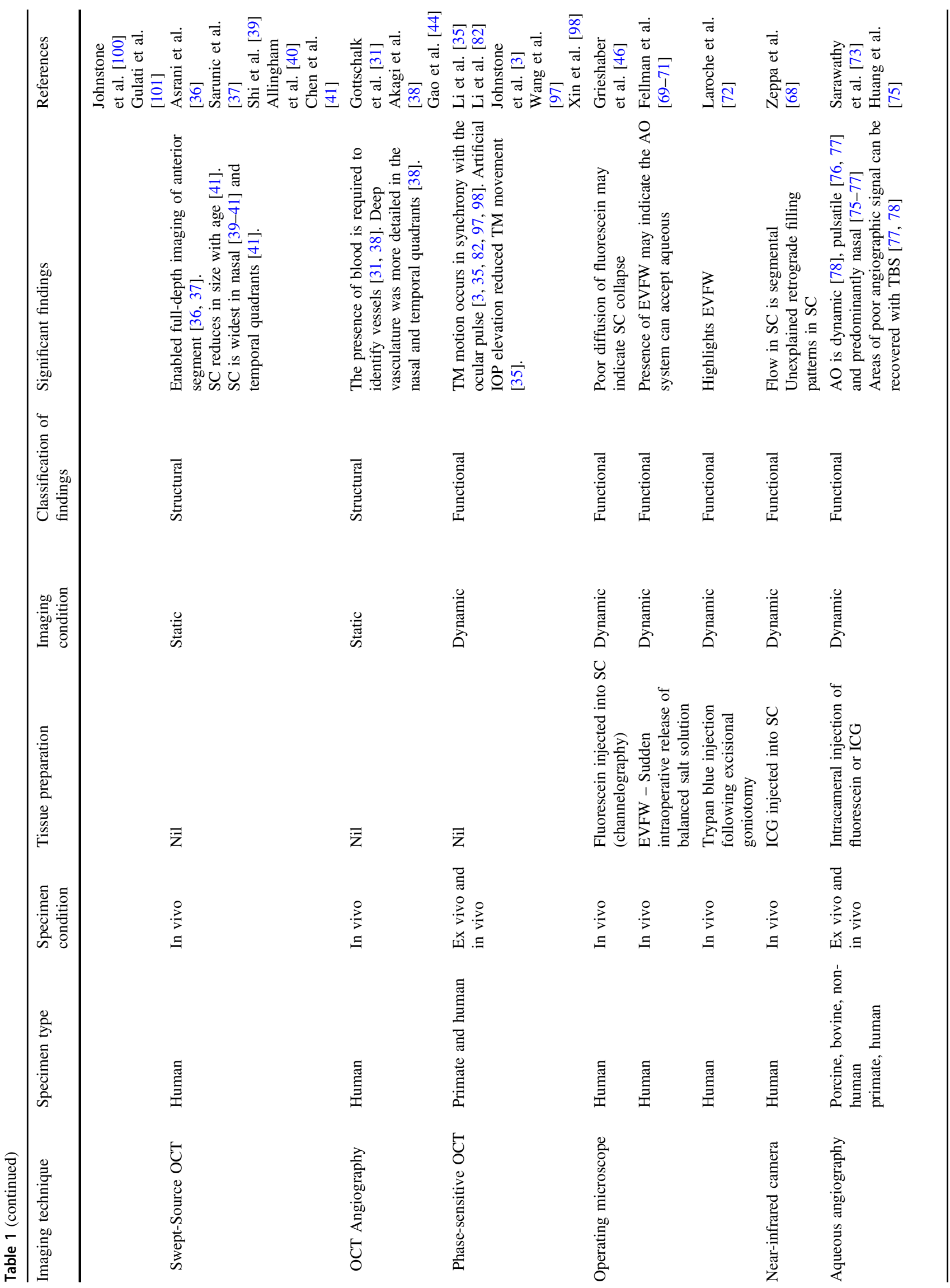




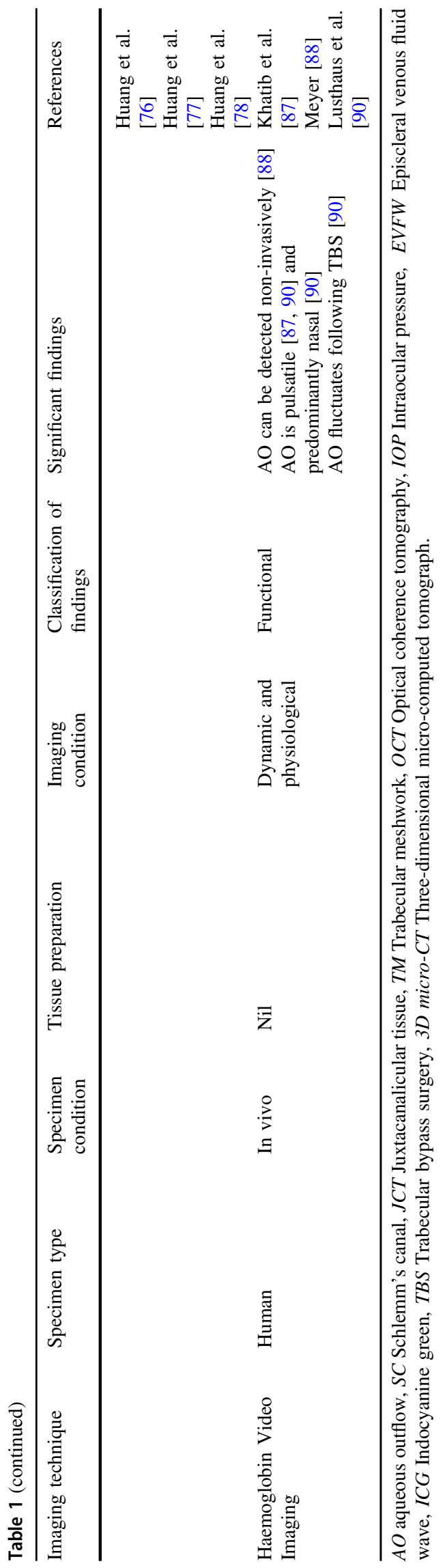

foundation of major theories of $\mathrm{AO}$ regulation that will be discussed later.

\section{Ribbon scanning confocal microscopy}

Ribbon scanning confocal microscopy (RSCM) was initially developed to speed up and improve the quality of brain tissue imaging [22]. It is derived from similar principles seen in confocal microscopy; where a laser is used to evoke the emission of fluorescent light from a defined depth within a sample. Single pinhole-focused images are generated and stitched together to form an overall image of a specimen. Instead of imaging multiple points, RSCM sweeps across specimens and creates image "ribbons" that are more easily stitched together and with less artefact [22]. As a result, imaging and processing occur ten times faster, and 3D reconstructions are possible (Fig. 3a) [23].

Imaging techniques such as spectral-domain optical coherence tomography (SD-OCT) are unable to detect small networks of vessels within the AO tract [24]. RSCM shows these networks in porcine eyes labelled with lectin via an intracameral bolus [25]. Dense tubular networks and lumens within the TM have been demonstrated, along with uninterrupted vessel branches from the TM to the scleral venous plexus, likely representing aqueous veins [25]. Collector channels were larger and more often seen within the nasal hemisphere where the greatest outflow is thought to occur [25]. Studies using RSCM in porcine and human eyes show anatomical differences. Human eyes have larger and longer collector channels than porcine eyes, but they are fewer in number [23].

\section{Two-photon microscopy imaging}

Trans-scleral visualisation of the AO system was demonstrated in ex vivo rat eyes by two-photon autofluorescence microscopy [4, 26]. Autofluorescence avoids the use of external fluorescent markers that may trigger tissue damage [26]. Initially, two-photon microscopy required surgical exposure to visualise SC. The subsequent use of additional filters refined the emitted signal and reduced the impact from scleral light scatter, enabling deep tissue imaging [27]. This method prevents the use of tissue fixatives that are often required in tracer studies and may cause tissue distortion [26]. A stack of generated images is reconstructed to form images of the TM, SC and aqueous veins [4, 26]. The AO tract was able to be reconstructed in 3-dimensions using specialised software that generated measurements of vessel lumens [4]. In addition, the cells within aqueous drainage tissues were labelled and profiled to identify their actions. Simulated IOP elevations have been shown by two-photon microscopy to cause local tissue damage to the TM and surrounding tissues [26]. Shifting from simulated results in 


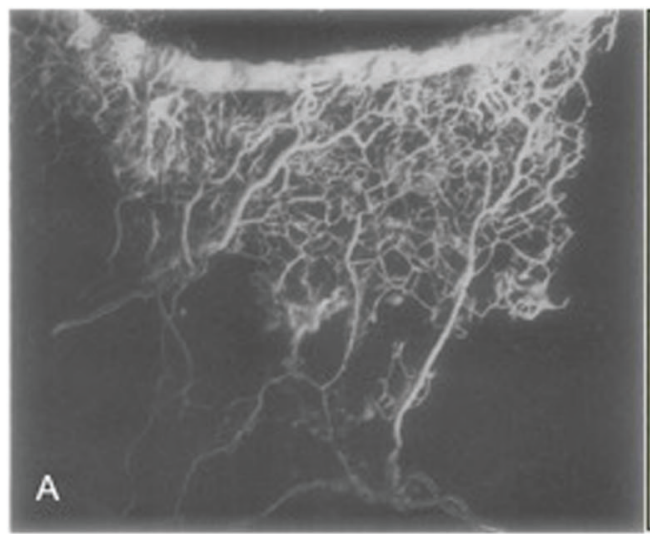

Fig. 1 A neoprene cast of human aqueous vein pathways. a Microscopic photograph of neoprene cast representing Schlemm's canal and associated distal aqueous outflow system. b Drawing of neoprene cast seen in a with green vessels representing the deep scleral

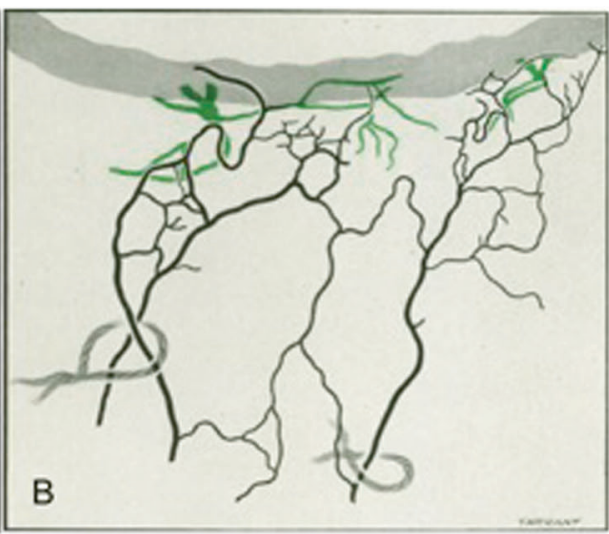

plexus and suture loops identifying aqueous veins. Both figures reproduced from Anatomical study of Schlemm's canal and aqueous veins by means of neoprene casts, Ashton N, 35, 291-303, (C1951 with permission from BMJ Publishing Group Ltd.
Fig. 2 Scanning electron microscopy image demonstrating the outflow pathway of aqueous (blue arrows) in a primate eye. $\mathrm{AC}$ anterior chamber, TM trabecular meshwork, ISCC intrascleral collector channels, AV-E episcleral aqueous vein, AV-C conjunctival aqueous vein. Image reproduced with permission from Johnstone, MA. Intraocular pressure control through linked trabecular meshwork and collector channel motion. In: Samples, JR., Knepper, PA., editors. Glaucoma Research and Clinical Advances: 2016 to 2018. Kugler Publications; Amsterdam; (2) 2016.

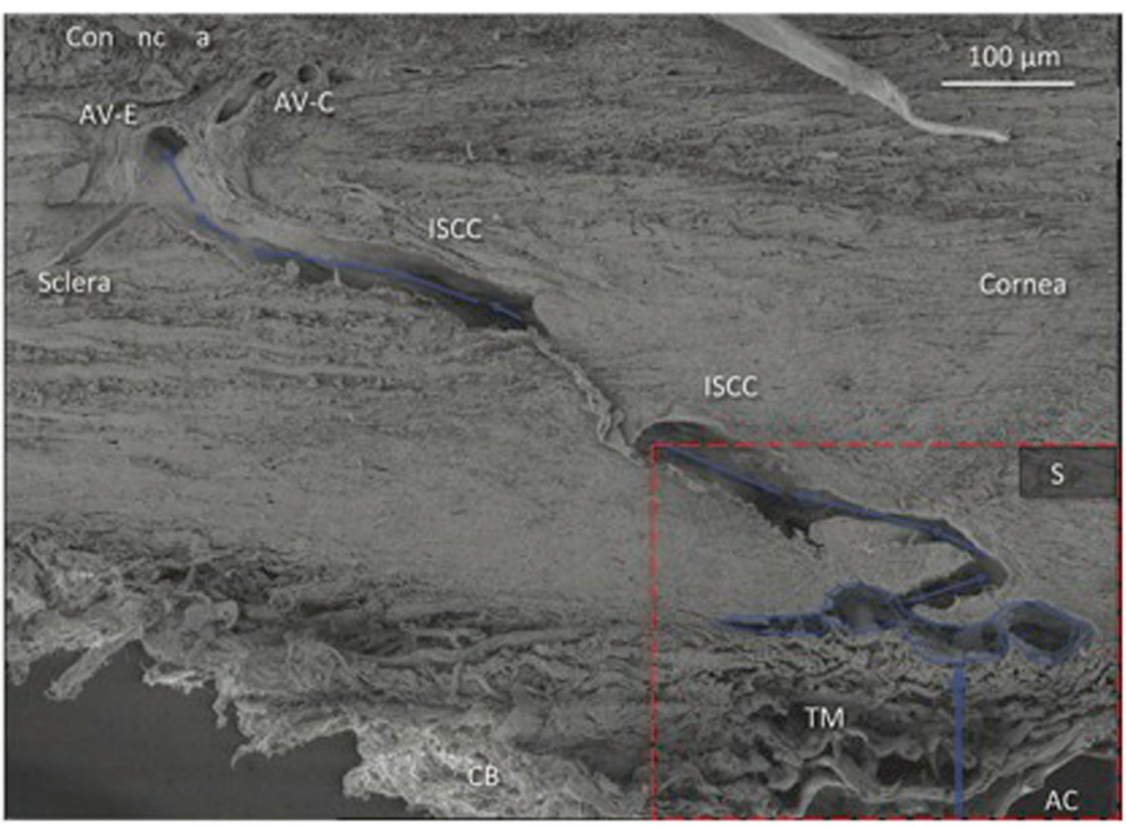

rodent eyes to modelling of the human AO pathway requires further refinement.

\section{Three-dimensional micro-computed tomography}

Many ex vivo imaging techniques produce data in sections or fragments that need to be reconstructed to represent the entire AO system. Conversely, three-dimensional microcomputed tomography ( $3 \mathrm{D}$ micro-CT) is a technique that generates an image of the whole anterior segment including TM, SC, collector channels and episcleral vasculature (Fig. 3b) [28]. Ex vivo human eyes were fixated with immersion or perfusion techniques and mounted on a rotating device. A molybdenum X-ray scanner was used to collect raw data that was then converted to allow 3D reconstruction and analysis [28, 29]. Osmium tetroxide $1 \%$ was used as a contrast agent to improve X-ray quality. Measurements of some AO components were possible, but perfusion fixation may have artificially opened flow pathways [28]. Ex vivo tissue processing can cause collapse of smaller vessels, which may explain the absence of deep scleral plexus vessels, when compared to neoprene casts [28].

\section{Spectral-domain optical coherence tomography}

The anatomy of the AO system is not amenable to standard optical coherence tomography (OCT) imaging. The 

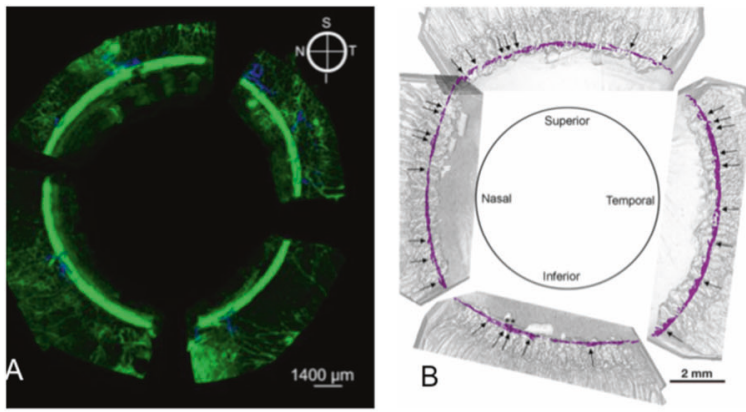

Fig. 3 Three-dimensional reconstructions of the human aqueous outflow system using multiple structural imaging techniques. a Ribbon-scanning confocal microscopy (collector channels in blue). b 3D micro-CT reconstruction demonstrating Schlemm's canal (purple ring) with narrowing superiorly in a perfused eye. Black arrows represent sites of collector channels evenly distributed throughout the circumference of the eye. c Virtual casting reconstruction of collector channels from an actively perfused in situ human eye imaged with SD-

episcleral vasculature is complex and difficult to resolve due to light scattering from the surrounding sclera [30, 31]. Despite this, the advancement of OCT technology made non-invasive imaging of the AO pathway possible for the first time. Spectral-Domain OCT (SD-OCT), with the assistance of Doppler signals, demonstrated the area of SC was larger in the nasal hemisphere compared to the temporal hemisphere. In a small number of subjects, patients with glaucoma had a smaller SC area than controls [32]. No evidence of SC or collector channels could be seen in one subject who had a functioning glaucoma drainage device. However, complete $360^{\circ}$ imaging of SC was not possible in this case, because images were taken in cross-section and blood vessels caused shadowing artefact [32]. Developments in image processing have led to complete reconstructions of the AO system in both ex vivo and in vivo human eyes using virtual casting (Fig. 3c) [33]. However, vasculature appeared less distinct in vivo, and vessels less than $10 \mu \mathrm{m}$ were poorly-resolved. In addition, arteries and veins could not be reliably differentiated [33].

Novel techniques incorporating OCT have been implemented to minimise image degradation, and to further characterize the relationship between TM and SC. An endoscope was able to image collector channels from within SC in ex vivo human eyes. The SS-OCT images were comparable to subsequent images taken with SEM [34]. Similarly, direct imaging of TM and SC through the anterior chamber produced comparable images to SEM. The addition of concurrent fluid pulses forced through SC allowed visualisation of how the TM responded to pressure changes [35].

\section{Swept-source optical coherence tomography}

Successful visualisation of the AO system requires highresolution imaging due to the complexity of the anatomy.

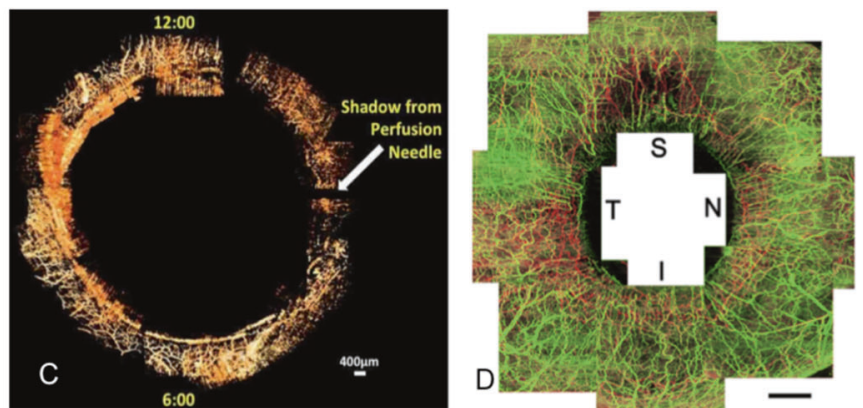

OCT. d OCT-A reconstructed image of merged superficial (green) and deep (red) vascular networks. Aqueous-carrying vessels are not identifiable. a Adapted image reprinted from Loewen et al. [22]. with permission under the terms of the CC BY license (https://crea tivecommons.org/licenses/by/4.0/). b Adapted image reprinted from [28], with permission from Elsevier. c Adapted image reprinted from [33], with permission from Elsevier. d Adapted image reprinted from [38], with permission from Elsevier.

Advancements in OCT technology have achieved resolutions in the tens of micrometres and made full-depth imaging of the anterior segment possible [36-38]. Swept-source OCT (SS-OCT) has demonstrated the structures of the human drainage angle, as well as the post-trabecular AO pathway within a single B-scan image (Fig. 4) [30]. Validation of SS-OCT has been made with comparison to histological specimens [39]. Vasculature within the episclera and sclera is complex and pathways of vessels are easily lost within a single B-scan. A technique used in computed tomography (CT) scanning, multiplanar reconstruction, provides a solution to this by compiling a continuous crosssection image of the area of interest [30]. The path of vessels can be studied using curved multiplanar reconstruction, a technique that involves re-slicing three-dimensional data. This has been applied to SS-OCT to generate a two-dimensional reconstructed image of the pathway between SC and the episcleral venous plexus [30]. However, it may be difficult to differentiate between vessels carrying aqueous and blood.

Measurements of SC area and its perimeter obtained with SS-OCT are comparable to estimates generated by human perfusion and histological studies [36, 40]. Chen et al. [41] used a commercially available SS-OCT (CASIA SS-1000; Tomey Corporation, Nagoya, Japan) to demonstrate a reduction in the diameter and area of SC with ageing [41]. Variations within SC were seen, the widest measurements being in the nasal and temporal quadrants. Increasing age correlated with TM thickening in the nasal and temporal quadrants [41]. SC was visible in $80.6 \%$ of eyes between 60 and 83 years of age. The authors suggest that reduced SC size and detection rates may result from tissue degeneration or shrinkage, a decrease in aqueous production with age, or distortion of the corneoscleral contour due to loss of ciliary muscle movement [41]. Optimisation of scan accuracy is 


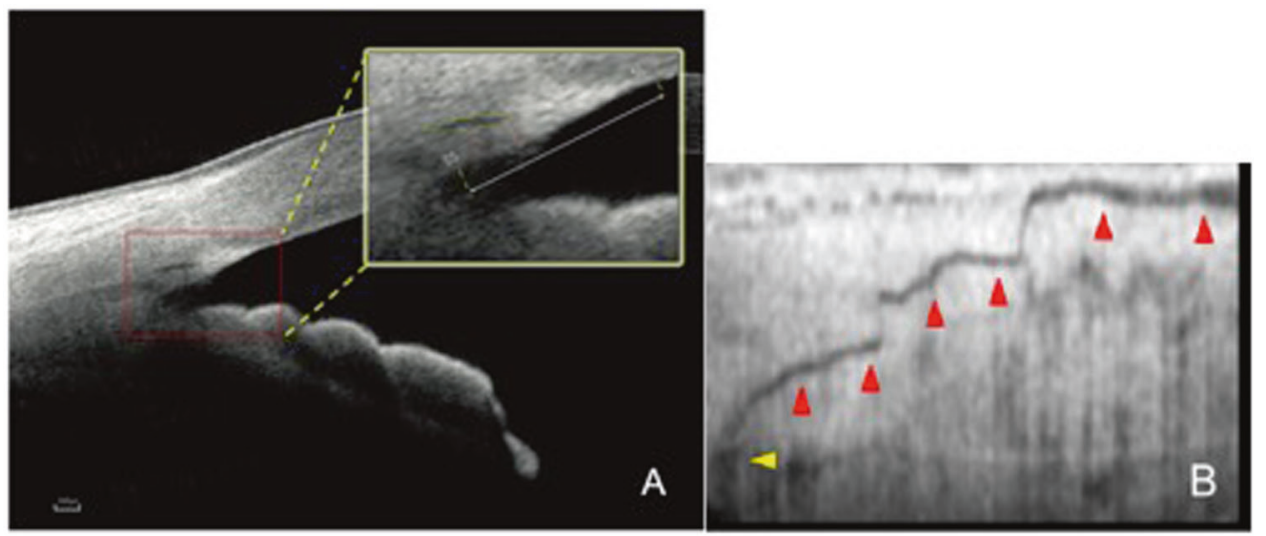

Fig. 4 Visualisation of the aqueous outflow system using sweptsource optical coherence tomography. a Swept-source optical coherence tomography (SS-OCT) of the human drainage angle. Trabecular meshwork and Schlemm's canal (black arrow) are pictured within the magnified box. b A single B-scan demonstrating the pathway of a post-trabecular aqueous outflow vessel (red arrowheads) emanating from Schlemm's canal (yellow arrowhead). The image was reconstructed using SS-OCT with flattening, en face imaging and reslicing techniques [30]. a Adapted image reprinted from Chen et al. [41] with permission under the terms of the CC BY license (https:// creativecommons.org/licenses/by/4.0/). b Adapted image republished with permission of Association for Research in Vision \& Ophthalmology (ARVO) from [30]. required to completely validate this technique [39]. Images are taken with the patient gazing to the opposite direction of the target quadrant, and not in primary position. There may also be diurnal variations in the morphology of SC that have not been identified in the current studies [41].

Swept-source OCT has been used to reconstruct images of the AO pathway before and after Trabectome and 360degree suture trabeculotomy, techniques used to reduce $\mathrm{AO}$ resistance [42]. No significant structural changes were detected as a result of these surgical techniques, despite good visualisation of the AO pathway [42]. The ability to compare pre- and post-operative SS-OCT data for an individual is challenging. Variability in diurnal AO and focus quality, as well as difficulty identifying the corresponding pre-operative AO pathway are limitations identified by the authors of this technique [42]. In another study, a 90\% increase in SC area within the nasal and temporal quadrants was demonstrated 8 $\mathrm{h}$ after instillation of travoprost $0.004 \%$ eye drops [43]. However, similar difficulties arose with identifying the corresponding pre-intervention site for comparison.

\section{Optical coherence tomography angiography}

The introduction of OCT angiography (OCT-A) has enabled the detection of blood flow within vessels of in vivo human eyes [38, 44]. Aqueous is more challenging to visualise with OCT-A than blood, so concurrent use of contrast is required. Akagi et al. [38] compared OCT-A with fluorescein scleral angiography and aqueous angiography using indocyanine green (ICG) [38]. En face OCT-A images at varying depths were reconstructed to represent the circumferential flow of superficial conjunctival vessels (>
$200 \mu \mathrm{m})$ and deeper intrascleral vessels (200 to $1000 \mu \mathrm{m})$ (Fig. 3d) [38]. The deeper vasculature represents the episcleral and intrascleral vessels responsible for draining aqueous from SC. Deep OCT-A vessel patterns reasonably correlated reasonably well with those generated by aqueous angiography, but further work is required to refine the technique into a reliable clinical tool.

Many aqueous-carrying vessels also contain red blood cells [1]. OCT-A will detect these vessels as long as there is concurrent blood flow $[31,38]$, but will not detect vessels containing only aqueous. Contrasts are used to improve visualisation with many other imaging modalities, but there is no dedicated contrast for OCT-A [31]. Gold nanorods have been shown to generate measurable Doppler OCT signals [45] and lipid emulsions (milk and propofol) induced strong OCT-A signals [31]. The findings from both of these studies are limited to ex vivo porcine eyes [31,45] OCT contrast studies are yet to be undertaken in human eyes.

\section{Functional aqueous outflow imaging}

Imaging techniques are evolving to encompass the dynamic nature of aqueous outflow. However, little is known about the physiology of AO distal to the trabecular meshwork and how drainage of aqueous is affected in glaucoma [25, 46]. Further study is warranted because the episcleral venous system is likely to be responsible for $25-50 \%$ of $\mathrm{AO}$ resistance [18, 47]. Visualisation of aqueous drainage is challenging and traditionally has been made possible with dyes such as fluorescein [46]. Recent imaging advances have facilitated the analysis of functional AO. 


\section{Fluorophotometry and tracers}

The imaging techniques discussed thus far provide structural representations of the AO system, but they are unable to demonstrate functional flow. Early attempts to study aqueous flow originate from fluorophotometry, a technique to measure aqueous concentrations of fluorescein in animal and human eyes [48-50]. Fluorescein may enter the anterior chamber of the eye via intravenous, oral or topical eye drop administration [50-52]. Disappearance of fluorescein from the anterior chamber is measured over time to reflect the rate of aqueous exchange. Multiple studies have recorded the rate of bulk aqueous flow in normal human subjects to be 1.6-3.4 $\mu \mathrm{l} / \mathrm{min}$ [52]. However, fluorescein clearance can be affected by physiological states, such as pupil dilation. Some fluorescein may be lost posteriorly in the eye and that may impact aqueous flow calculations [53]. In addition, direct visualisation of fluorescein drainage from the eye is not possible with this technique.

A variety of tracers have been used to study aqueous drainage in more detail; particularly, to identify flow variations around the circumference of the eye and anatomical responses to varying perfusion pressures [54]. Cationic ferritin, gold particles, fluorescent microspheres and nanospheres, latex beads and quantum dots have all been used as tracers [54-61]. Most of these studies perfuse ex vivo animal or human eyes with tracer. Confocal, light, or electron microscopy techniques are then used to generate images of the labelled and processed tissue sections [54-61]. These techniques rely on tissue adherence or active cellular uptake of tracers to help identify structures within the AO pathway. Tracers have a larger molecular weight than water, and so they accumulate at the TM and collector channels [8].

Segmental labelling of the TM in multiple studies suggests regions of preferential aqueous outflow in all eyes [54, 58, 59, 61]. However, one study demonstrated homogenous cationic ferritin labelling in normal eyes compared to segmental patterns of uptake in glaucomatous eyes [62]. Further work in healthy ex vivo human eyes suggests preferential outflow where pigmented TM is in close proximity to collector channels [61]. Tracer imaging has also been used to illustrate the IOP-lowering mechanisms of Rho kinase inhibitors, which include TM and JCT expansion, and vasodilation of episcleral veins [56]. Despite the significance of the findings from tracer studies, there are a number of limitations due to the use of ex vivo eyes. Conditions are not physiological and do not account for other variables such as; aqueous production, ciliary body tone, EVP and uveoscleral drainage [56]. Processing of histological sections is required to assess tracer response, which makes this imaging technique incompatible with studying in vivo human eyes [8]. Real-time analysis of AO

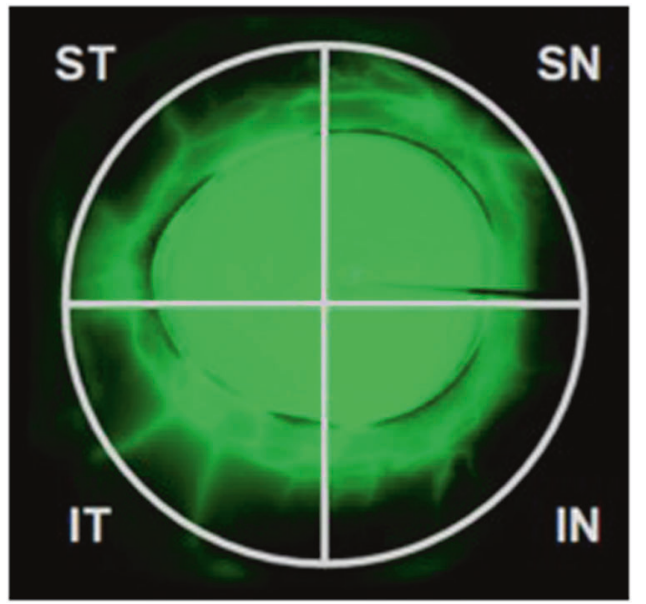

Fig. 5 Fluorescein canalogram taken from a perfused whole porcine eye. Adapted image reprinted from Loewen et al. [63] with permission under the terms of the CC BY license (https://crea tivecommons.org/licenses/by/4.0/).

is required to best reflect the pathophysiology of IOP dysregulation as seen in glaucoma.

A similar technique, canalography, is another form of tracer study, but histological section is not required. Fluorescent microspheres have been injected into the anterior chambers of ex vivo porcine eyes and visualised using a dissecting microscope capable of fluorescence imaging [63, 64]. In studies of non-glaucomatous porcine eyes, canalograms have demonstrated regional and circumferential outflow patterns with an increase in fluorescence nasally (Fig. 5) [63]. This finding was accentuated following $a b$ interno trabeculectomy, but improvement of flow and peak fluorescence was seen in all quadrants [64]. Another ex vivo porcine study used canalography to demonstrate the effects of trabectome-mediated ab interno trabeculectomy and trabecular micro-bypass implantation [65]. Fluorescein perfusion was initially administered to identify $\mathrm{AO}$, and then Texas red was perfused postprocedure to demonstrate the effects of each surgery. Both surgical techniques achieved a regional improvement in $\mathrm{AO}$ [65]. These studies provide validation for surgically reducing trabecular resistance, but limitations of the use of ex vivo primate eyes prevent comparison with what occurs in the episcleral venous system.

\section{Intra-operative techniques}

\section{Channelography}

Surgical access to SC using techniques such as viscocanulostomy and canaloplasty have created opportunities to image the AO system more clearly. Viscocanulostomy is achieved by dilating SC with a viscoelastic [66], whereas canaloplasty includes the additional step of leaving behind 

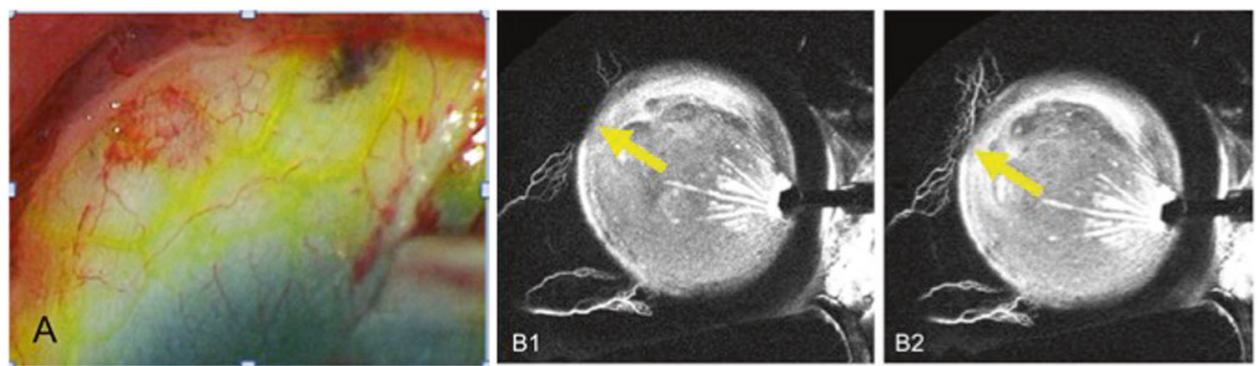

Fig. 6 Intra-operative techniques to visualise distal aqueous drainage and immediately confirm surgical success. a Fluorescein-filled episcleral vessels after injection directly into Schlemm's canal. b Aqueous angiography demonstrating increasing signal in the superonasal quadrant during cataract surgery. Images are taken 176 seconds (B1) and 182 (B2) seconds after introduction of $0.04 \%$ indocyanine green. a Reprinted by permission from Springer Nature. Ophthalmologe. Grieshaber MC. Kanalographie und Wirkmechanismus der Kanaloplastik [Channelography and mechanism of action in canaloplasty]. 112(4):319-324 @2015. https://doi.org/10.1007/s00347-0143163-4. b Adapted image reprinted from [8] with permission from John Wiley and Sons. an intracanalicular suture thread to maintain SC [67]. The goal of both techniques is to reduce $\mathrm{AO}$ resistance by expanding SC, however, the success of these surgical techniques depends on the functionality of the distal $\mathrm{AO}$ system [46]. The desire to improve surgical success rates has led to further steps being added at the beginning of the surgery to ensure AO patency. Provocative gonioscopy or ocular decompression cause blood to reflux into SC, and this can be easily visualised with an intraoperative gonioscope. Fluorescein may be injected from a microcatheter into SC to enable downstream visualisation within the EVS and reflux into the anterior chamber (Fig. 6a) [46, 67]. The latter technique became known as channelography, in which fluorescein is directly visualised with the operating microscope [67]. The health and permeability of the TM may be represented by the amount of fluorescein that leaks into the anterior chamber [46]. Similarly, a greater number of episcleral vessels that accept fluorescein within an eye may reflect superior functional capacity within the distal drainage system [46]. However, channelography bypasses outflow resistance generated by the TM and requires manual perfusion of contrast directly into SC [8]. Both of these factors mean that patterns of outflow are non-physiological and do not replicate aqueous outflow, despite providing clear images of the outflow tracts.

Indocyanine green (ICG) channelography has been achieved with a near-infrared camera in human in vivo eyes [68]. The choice to use ICG combined with viscoelastic was to prevent excessive leakage from SC and collateral fluorescence. In some eyes, areas of SC appeared non-patent as evidenced by incomplete fluorescence. Collector channels only filled in corresponding areas of SC patency, suggesting possible intracanalicular obstruction [68]. Increasing the perfusion pressure and volume enabled visualisation of collector channels not previously evident. Retrograde filling by ICG of glomerular patterns within the ring of SC were not fully explained. The authors state that the structures did not leak and suggest they may form part of the iris or even the lymphatic system [68]. Although there is no clear explanation for these structures, it is conceivable that they, or potentially other undetected structures, contribute to IOP regulation.

\section{Operating microscope}

Surgical intervention provides a unique opportunity to study the AO system and the effects of procedures that target the trabecular meshwork. Variable MIGS results have driven studies that particularly focus on advancements in real-time image acquisition and $\mathrm{AO}$ visualisation. A number of intraoperative techniques have been reported that help evaluate aqueous drainage. In one technique, known as episcleral venous fluid wave (EVFW), a pulse of balanced salt solution was suddenly released by the irrigation and aspiration hand-piece to examine the patency of the distal episcleral vessels following cataract surgery combined with trabectome (ab interno trabeculotomy) [69]. The authors postulate that the presence of an EVFW indicates that the episcleral venous system is able to accept aqueous. Conversely, the absence of an EVFW may predict a higher chance of surgical failure, possibly due to a blockage, stenosis or atrophy within the system [69]. In a retrospective study, the extent of EVFW around the circumference of the eye inversely correlated with IOP in the short-term postoperative period [70]. Eyes with poor or no visible EVFW were associated with a poor trabectome result and a onethird chance of requiring further glaucoma surgery [70]. Furthermore, the extent of EVFW was compared between trabectome and iStent, and both were found to generate segmental flow improvement [71]. Trypan blue has been used to highlight flow within the EVS following ab interno TM excision with the Kahook Dual Blade (New World Medical, Rancho Cucamonga, CA) [72]. This technique essentially improves visualisation of the EVFW. Whilst the 
detection of EVFW and its extent may help to assess surgical success or prognosis on the operating table, it does not reflect true physiological drainage or functionality of the AO system. The potential effects of phacoemulsification on $\mathrm{AO}$ are also not addressed.

\section{Aqueous angiography}

Another intraoperative imaging technique, aqueous angiography (Fig. 6b), was developed to enhance visualisation of AO pathways in real-time. Initial work was on ex vivo porcine [73], bovine [74] and human eyes [73, 75], but intraoperative studies on in vivo non-human primate eyes [76] and then human eyes [77] soon followed. Aqueous angiography incorporates steps from a number of imaging techniques that have already been discussed. It takes advantage of intraoperative access to the proximal aqueous drainage structures via the anterior chamber, and incorporates a tracer that can be externally detected with a bespoke angiographic imaging system. A specialised arm supports the angiography equipment (Spectralis HRA + OCT Flex module; Heidelberg Engineering, Heidelberg, Germany) above the patient's eye, which is centred with the assistance of confocal scanning laser ophthalmoscopic infrared images [76-79]. The anterior chamber is supported by a continuous infusion whilst a fluorophore is introduced intracamerally. Although the anterior chamber dynamics are non-physiological, this method examines the functionality of the TM and subsequent flow into distal outflow channels. Angiographic images are recorded as the patient is asked to rotate the eyes in various directions to avoid obstruction by the eyelids. This technique enables real-time assessment of circumferential aqueous drainage in live human eyes [7779].

Both ICG and fluorescein have been safely administered as intracameral tracers. Sequential use of tracers (ICG first) is possible and yields similar results $[74,75,78,79]$. This permits angiography to demonstrate the flow response before, and immediately after, an intervention. Aqueous angiography has been applied to support established AO anatomy findings, identify spontaneous dynamic changes within the AO system, and to demonstrate that areas with poor angiographic signal can be recovered with trabecular bypass surgery [77, 78]. Although aqueous angiography comes closer to visualising physiological AO than other imaging techniques there are still a number of limitations that prevent this goal from being achieved. It is an invasive process and requires patient sedation, pupil dilation and topical anaesthesia prior to surgery, all of which are likely to affect AO and IOP. The use of an intraoperative speculum and anterior chamber maintainer alter IOP and aqueous dynamics [77]. Fluctuations in IOP during surgery also affect aqueous dynamics and imaging [78]. Despite these limitations, aqueous angiography has contributed significantly to our understanding of the AO system and how MIGS can interact with AO. Nevertheless, non-invasive imaging is required to assess physiological AO.

\section{Functional imaging in the clinic}

\section{Phase-sensitive optical coherence tomography}

Pulsatile AO was initially reported by Ascher [80] and was thought to originate from SC in synchrony with ocular pulsation [80, 81]. The static nature of OCT and OCT-A is a significant limitation when imaging dynamic aspects of the $\mathrm{AO}$ system. $\mathrm{Li}$ et al. [35, 82] described a novel OCT technique to enable assessment of TM motion in living human eyes $[35,82]$. Phase-sensitive OCT (PhS-OCT) is able to quantify tissue motion to the nanometre, a measurement not possible with traditional OCT [35]. The technology is derived from the use of Doppler OCT to measure blood flow [83]. Each standard OCT B scan contains $500 \mathrm{~A}$ scans, and it is the measurement of the phase changes between successive A scans that reflect tissue movement [83, 84]. IOP variations were generated in an ex vivo primate model to simulate ocular pulsations. The TM was displaced towards SC in synchrony with each generated pulsation. Artificial elevation of IOP reduced TM displacement and SC area was reduced [35]. PhS-OCT has advanced to permit imaging of human subjects on a system similar to a regular outpatient slit-lamp. This technology has been used to support the findings of Johnstone et al. [3, 85] who identified a model of IOP regulation controlled by pulse-dependent TM motion [3, 85].

\section{Haemoglobin video Imaging}

Until recently, imaging of conventional aqueous outflow pathways has required the introduction of a marker into the anterior chamber. However, Haemoglobin Video Imaging (HVI), a slit-lamp technique originally developed to investigate limbal microvascular blood flow [86], can demonstrate these pathways without the need for more invasive methods [86, 87].

HVI exploits the absorption spectrum of haemoglobin to demonstrate red blood cells in the conjunctival and episcleral circulations [86, 88, 89], and can illustrate wherever venous blood is parted by clear cylindrical columns of aqueous: "aqueous veins".

The circulations are illuminated by a broad bandwidth $(540-580 \mathrm{~nm})$, which corresponds with the longwavelength absorption peak of haemoglobin, causing erythrocytes to appear dark against the background of bright, reflective sclera. In contrast, aqueous is seen as a paraxial, translucent lamina within the blood column. 

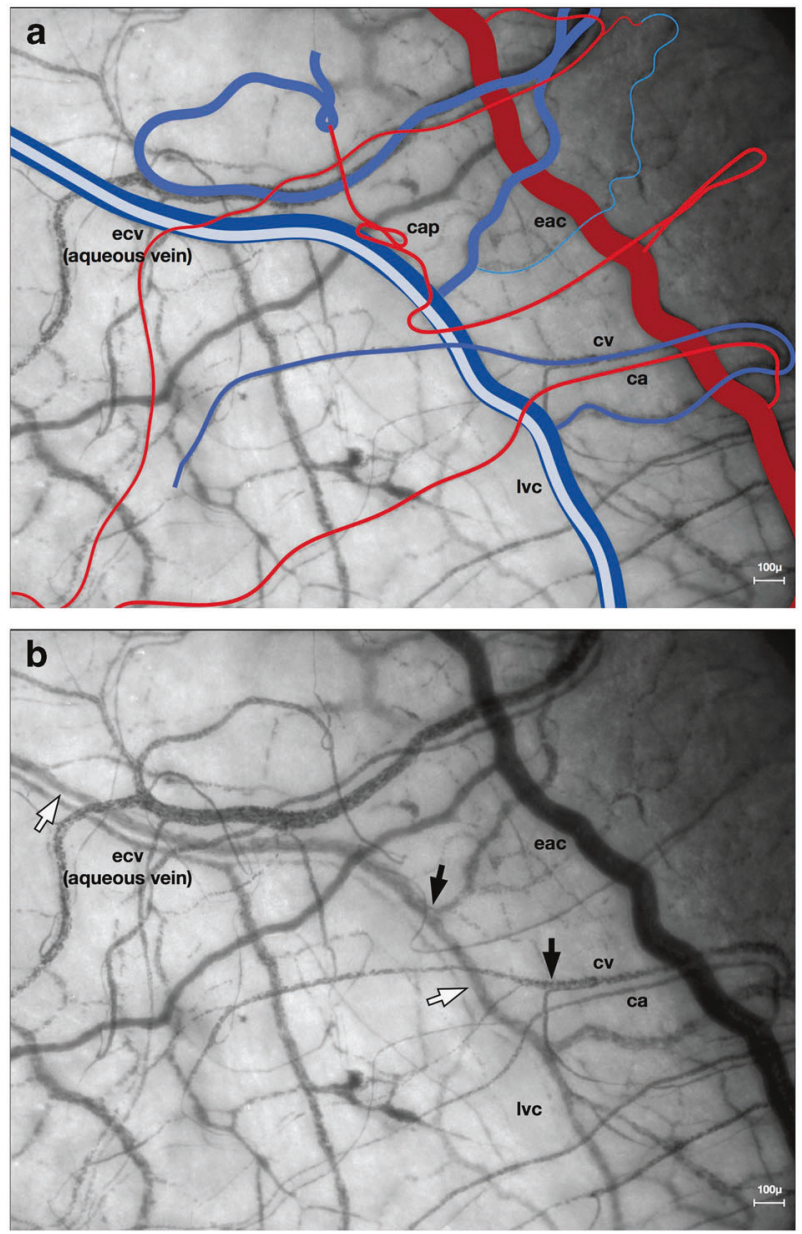

Fig. 7 Aqueous drainage within ocular surface microcirculations. a, b Anterior segment microcirculations. eac episcleral arterial circle, lvc limbal venous circle, ecv episcleral collecting vein, ca conjunctival arteriole, cv conjunctival venule, cap capillary. b The black arrows demonstrate blood striation and the white arrows identify an aqueous column.

The images are captured with a sensitive, high-resolution, monochrome video camera and bespoke stabilisation software eliminates motion artefact due to micro-saccades [88].

Patients are examined with HVI in a darkened clinic room and are positioned as they would be for standard slit lamp examination. The entire limbus can be recorded by asking the patient to adjust fixation.

Familiarity with the ocular surface microcirculations [89] is helpful to differentiate their complex hierarchy (Fig. 7). In general, vessels delivering blood to microcirculations tend to be more tortuous, sometimes with intermittency of flow, and they may demonstrate advancing constrictions. Blood in drainage vessels appears granular, and converging venules add successive laminae to the blood column.

The smallest vessels in the episcleral circulation often form an interconnecting lattice and appear larger than conjunctival capillaries. Constrictions are not visible and

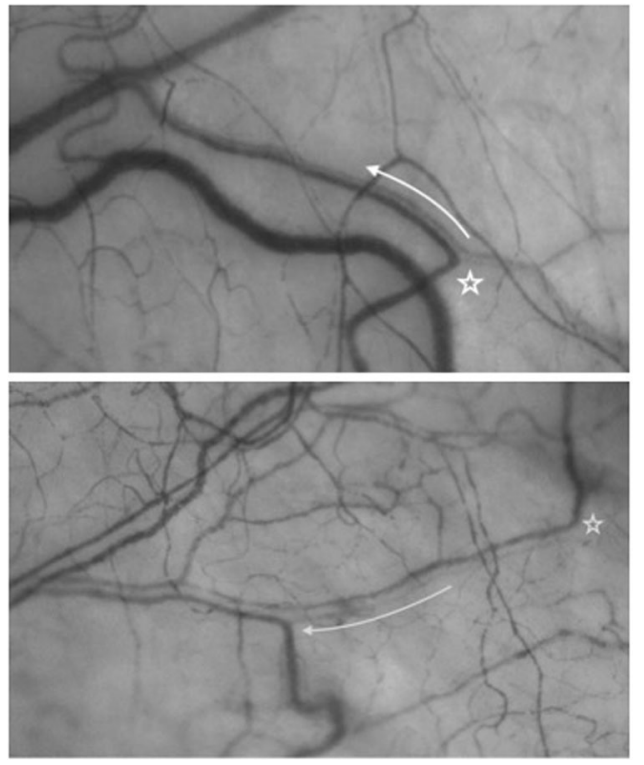

Fig. 8 Two examples in which blood and aqueous drain into a common vessel to create an asymmetrical flow pattern. The points where blood and aqueous combine are marked with white stars. The direction of distal aqueous flow is indicated by the white arrows.

flow may freely reverse. Perfusion of any segment of the ocular surface microcirculation may be periodic, erythrocytes remaining static for long periods [89].

Aqueous veins are a consequence of laminar flow within venous blood. As successive venous channels converge, they contribute to the periphery of the venous column, remaining discreet for considerable distances before they ultimately combine.

Since aqueous usually enters the venous system early in its course, aqueous laminae tend to lie centrally. However, when aqueous is added to an established blood column, it usually remains peripheral until displaced axially by subsequent contributions of venous blood (Fig. 8) [90].

Visualisation of aqueous using HVI was first reported in 1989 [86] and creates a unique opportunity to study physiological AO in living human eyes [89]. Although aqueous is detected indirectly, by the displacement of erythrocytes within episcleral collecting veins (Fig. 7b), a number of aqueous vein characteristics previously reported by Ascher [1] have been demonstrated [87, 90]. These include: pulsatile flow corresponding with the heart rate, parallel stratification of flowing blood and aqueous, disruption of the aqueous stream during eye movements and gentle globe compression, and redirection of aqueous flow into adjacent episcleral vessels following localised aqueous vein compression.

HVI has recently graduated from a qualitative to a quantitative modality [87] (Fig. 9a). In HVI, absorption of incident light increases with thickness of the erythrocyte barrier between the light source and sclera. Computer 

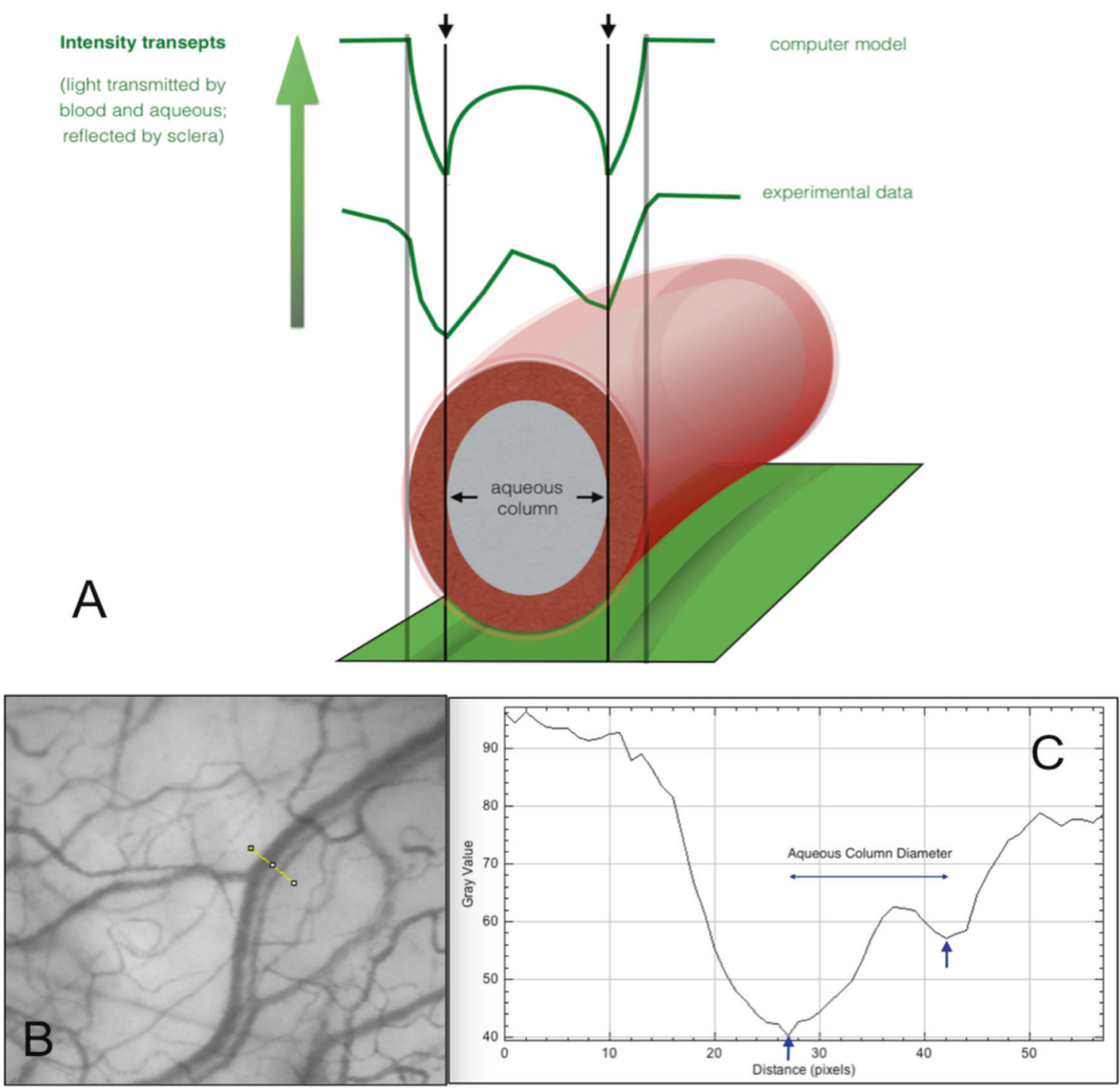

Fig. 9 The method used to calculate aqueous column crosssectional area. a Diagrammatic representation of aqueous column measurement from light intensity transepts. b, c An aqueous vein is pictured with a linear transept cutting across the vessel. The transept and corresponding graph are generated by Image $\mathrm{J}$ software. The aqueous column diameter equals the distance between the troughs (vertical blue arrows). The diameter in pixels is converted to aqueous column cross-sectional area (AqCA) in micrometres squared. modelling of the intensity of light absorbed by transepts of aqueous veins demonstrates that the transition between blood and aqueous corresponds with the transmission minima. Therefore, from light intensity transepts (ImageJopen source software), it is possible to calculate aqueous column cross-section area (AqCA), a statistic presumed to be proportional to bulk flow in the cylindrical aqueous column [87, 90] (Fig. 9b, c). At least three transepts are measured and the calculations, averaged. Measurements of AqCA have been used to demonstrate improvements in $\mathrm{AO}$ following selective laser trabeculoplasty and trabecular bypass surgery (iStent Inject) [87, 90].

Although HVI has many potential clinical applications, its use in studying $\mathrm{AO}$ is in its infancy. At the time of writing, the technology has not yet developed into a turnkey ancillary test. Visualisation of AO within the EVS with HVI is straightforward, but quantification techniques are currently laborious and need to be streamlined. Measurements vary between vessels within the same eye, but the same vessel can easily be identified in successive scans for comparison and to assess the effects of interventions. A normative database is not yet available for AqCA and further work is required to establish diurnal variations in aqueous flow. Despite this, HVI is the only aqueous outflow imaging system that can generate anatomical and flow data non-invasively in physiological conditions in human eyes in vivo. The proximal drainage structures, TM and SS, cannot be seen with HVI, meaning complimentary imaging methods such as OCT will be needed to provide functional assessments of the entire outflow system. 
Future directions for HVI include three broad approaches. Firstly, the definition of normal AO is required to contextualise changes seen in varying levels of IOP and glaucoma severity. The purpose of this is to assist with glaucoma staging and it may lead to more detailed classification of open-angle glaucoma. Secondly, it is necessary to study the effects of medical, laser and surgical interventions on $\mathrm{AO}$ in order to identify factors associated with success, enabling a personalised approach to IOP-lowering treatment. In particular, information on the patency of the AO system may help to refine our choice of surgical tool. Or in the case of TBS, the location of device insertion may be optimized to improve IOP-lowering outcomes. Lastly, HVI creates an opportunity to observe, manipulate and study physiological aqueous drainage. HVI findings may advance the current understanding of mechanisms that control AO and IOP regulation.

\section{The role of imaging in theories of aqueous outflow regulation}

The eye is amenable to imaging and this has been exploited, particularly for retinal conditions. However, the AO system is a far greater challenge due to its complex and concealed pathways through varying densities of ocular tissue. Emerging technologies have exposed the anatomy and some functional aspects of the AO system. The presence of homeostatic mechanisms responsible for AO drainage and IOP control has been widely speculated in the literature for some time [2-6, 90]. It is the regulatory mechanism that continues to elude researchers and its discovery will likely revolutionise glaucoma diagnosis, prognosis and treatment. Imaging techniques that define structure have become noninvasive in the form of various types of OCT scanning, but functional AO assessment has, until recently, been invasive. Despite many challenges, imaging studies have provided researchers with critical information, leading to detailed theories of AO regulation. Our understanding of IOP homeostasis is likely to invoke evidence from all these imaging modalities.

\section{What have imaging studies taught us about IOP regulation?}

Static ex vivo and in vivo imaging studies have described the structure of the AO system in great detail. It is generally understood that aqueous flows from the anterior chamber through TM and into SC. From there, aqueous enters collector channels and flows into the deep scleral plexus. Intrascleral collector vessels drain aqueous into episcleral veins that flow into the ophthalmic veins, which traverse the orbit to join the cavernous sinus. More detail has been uncovered by recent imaging studies to begin explaining the intricacies of aqueous flow through this pathway and to test theories of AO. It is important to remember that histological data contribute considerably to our understanding, but are beyond the scope of this review.

\section{Limitations of the passive flow theory}

There is now a large volume of corroborative evidence to refute the theory that aqueous drainage through the conventional outflow pathway is purely passive [11, 20], down a pressure gradient [5]. Variations of flow patterns have been visualised throughout the system. Regions of preferential flow exist within the TM [54] and evidence of asymmetrical SC and EVS drainage patterns, as well as the discovery of valves within SC have been uncovered by AO imaging [54, 61, 67, 68, 71, 75-77, 90]. Intra-operative channelography and episcleral venography studies support findings from tracer studies that SC is discontinuous. Recurring anatomical findings suggest the eye is structured to drain aqueous predominantly in the nasal and inferior quadrants $[71,75-77,90,91]$. There is no specific pattern to the distribution of collector channels identified with 3D micro-CT [27], but variations in anatomy throughout the circumference of the eye have been demonstrated with multiple imaging techniques to indicate preferential drainage [25, 32, 39-41, 91]. RSCM identified that scleral venous plexus vessels receiving aqueous were larger in the inferior limbus and the largest collector channels were in the nasal quadrant [25]. SC diameter in living human eyes was shown to be larger in the nasal quadrant when imaged with S-D OCT [32] and SS-OCT [39-41]. 3D micro-CT showed that SC was attenuated superiorly, but appeared dilated at collector channel openings [28]. Deep vasculature was more dense and complex within the nasal and temporal quadrants than elsewhere, perhaps indicating sites of preferred $\mathrm{AO}$ [38]. However, anterior segment OCT-A identifies luminal structures regardless of their function, so it is difficult to draw definitive conclusions [38]. Infero-nasal AO has been shown to predominate in human flow studies [61, 68, 75$77,90]$. This information not only reassures surgeons to continue targeting the nasal quadrant when performing TBS, but also cultivates the notion that aqueous drainage may be regulated, rather than passively leaving the eye.

\section{The funneling theory}

The trabecular meshwork is thought to account for $\sim 50-75 \%$ of AO resistance in human eyes [11, 20, 22, 32]. The ultrastructural relationship of the JCT and SC endothelium has been visualised with electron microscopy [17]. Elastic fibres of the JCT lie tangentially to SC endothelial cells. This anatomical relationship may be responsible for baseline $\mathrm{TM}$ resistance to $\mathrm{AO}$, but the specific site of 
resistance continues to be debated in the scientific literature $[17,92]$. Out-pouchings of SC endothelium into the canal lumen are often referred to as 'giant vacuoles'. Electron microscopy has also detected pores at the distal end of vacuoles, which are thought to aid transcellular fluid movement [92]. The presence of these pores forms the basis of the 'Funneling theory' of AO [93, 94]. Essentially, aqueous is forced to drain preferentially through the JCT in regions where giant vacuoles and pores are present. Based on this theory; the JCT provides most of the resistance to $\mathrm{AO}$, but giant vacuoles and pores within SC endothelium can moderate resistance and flow [92-95].

The funneling theory is supported by the finding of asymmetrical and segmental trabecular outflow. Tracer studies confirm segmental flow through the TM with labelling of only 10-20\% of the SC inner wall [92]. Variations in TM thickness; specifically thickening of TM in regions of active outflow compared with inactive areas, and higher numbers of collector channels in regions of active flow, substantiate segmental AO [91]. Thickening of the TM may be due to JCT expansion. This has been highlighted by fluorescein bead deposition adjacent to collector channels in response to raised IOP in perfused ex vivo human eyes [61]. Although pores have been implicated in the control of outflow facility in a number of studies [96], the funneling theory is limited by the potential for tissue fixation processes to create artefactual pores [92]. There is also poor correlation between measured AO resistance and SC inner wall pore density in a number of studies [93].

\section{The mechanical pump theory}

Anatomical variations throughout the circumference of the anterior segment provide clues about how aqueous may flow through the conventional outflow system. However, real-time observation is required to appreciate the dynamic nature of the AO system and understand its physiology. Evidence of pulsatile aqueous flow in synchrony with cardiac pulse dates back to Asher's observations [1], and the phenomenon continues to be reported as imaging technologies develop [2, 3, 76, 77, 81, 88, 90]. The TM does not contain smooth muscle, but contractions may originate from nearby connections of ciliary muscle fibres [4]. Pulsatile aqueous flow has been observed in normal and glaucomatous eyes, but with reduced pulsatility in the latter group [3]. Some IOP-lowering medications have been shown to increase pulsatile AO [3]. More recently, TM movement, in synchrony with ocular pulse, has been demonstrated with PhS-OCT [35, 82, 97, 98]. This reflects the elastic properties of the TM and indicates its possible role in maintaining IOP homeostasis [5].

TM motion, as detected by $\mathrm{PhS}-\mathrm{OCT}$, provides evidence to support the 'mechanical pump' theory. The basis of the theory is that ocular pulse, blinking and sudden changes in eye position create transient increases in IOP that propagate through the AO system. Outward deformation of the TM causes SC narrowing and expulsion of aqueous into collector channels. As the pulse subsides, the TM rebounds to its initial position and SC widens. Aqueous from the anterior chamber then moves down a pressure gradient through valves to fill SC $[2,84,98]$. The TM may contribute to maintenance of IOP homeostasis by varying its compliance with each pulsation. Ongoing insults to TM integrity could possibly cause permanent anatomical and functional changes. For example, in glaucomatous eyes, a reduction in pulsatile aqueous flow may be caused by an increase of TM stiffness [6, 98]. Also, IOP changes have been shown to alter AO pulsatility. Ocular hypertension $(>28 \mathrm{mmHg})$ causes a reduction in visible pulsation [3]. It has been hypothesized that progressive apposition of SC walls due to TM movement during raised IOP increases AO resistance [5]. Temporary lowering of IOP below a physiological level also reduces pulsatile flow [3]. All of this evidence favours mechanical pumping of aqueous into collector channels, but other active drainage mechanisms are equally likely to be present within the distal outflow system. Despite the contribution of the TM, 25-50\% of residual AO resistance $[11,20,22,32]$ remains unexplained and must occur within $\mathrm{SC}$ or the distal outflow tract.

\section{The role of Schlemm's canal}

Schlemm's canal is a complex structure that was previously thought to act merely as a reservoir to collect aqueous from the anterior chamber and distribute it into aqueous veins [1]. Through ex vivo imaging studies we now understand that there are microstructures within SC that may influence AO control. Adhesions and septal columns have been visualised within areas of SC using scanning electron microscopy. They likely maintain patency of the canal and prevent inner wall tissue from causing herniating blockages into nearby collector channels [16]. In addition; a lumen within septal columns may facilitate one-way drainage of aqueous across SC to collector channel openings $[6,16]$. Valve-like structures have been identified at the collector channel openings, which may prevent backflow into SC $[5,16]$. The intraluminal structure of SC further supports an underlying mechanism behind AO regulation. Experimental manipulations of IOP and SC also demonstrate a relationship between the two. IOP was artificially elevated using an ophthalmodynamometer and S-D OCT imaged SC [99, 100]. Raised IOP reduced SC cross-sectional area by $32 \%$ in healthy eyes [99], compared to a $50 \%$ reduction in cadaver eyes [100]. Furthermore, increasing SC-CSA with an intraluminal scaffold caused a reduction in IOP [101]. These findings indicate that there is likely a baseline 
structural stiffness of SC inner wall that helps to maintain SC patency and IOP control. This creates a "chicken and egg" scenario where loss of TM and SC integrity over time (TM thickening and shrinking of SC) [41] may cause raised IOP, or pathologically raised IOP may cause loss of structural integrity.

\section{Potential for distal outflow regulation}

Further proof of the dynamic nature of aqueous drainage is seen with real-time aqueous angiography. Spontaneous variations in regions of outflow appear as increasing or decreasing angiographic signal [76, 77]. Similar AO fluctuations are seen under physiological conditions with HVI and are thought to be due to diurnal variation [90]. It is possible that these changes reflect homeostatic responses in the form of localised vessel wall contractions, control of flow through TM or SC, or both. An initial absence of flow does not necessarily reflect whether a vessel is able to accept aqueous [78]. This can be confirmed by applying localised pressure to the globe or directly to an aqueous vein. Aqueous flow is then directed into adjoining blood-filled vessels $[1,87]$. It is also possible that as structures, such as TM, SC or collector channels, become pathologically altered over time, flow is diverted towards healthier pathways within the system to reduce the burden and maintain IOP [61].

Recovery of AO in low flow regions has been achieved with TBS and verified with aqueous angiography [75, 78]. Furthermore, HVI has illustrated that TBS can re-establish $\mathrm{AO}$ in regions of the microcirculation previously devoid of aqueous [90]. The non-invasive nature of HVI enabled serial follow-up of post-operative TBS (iStent Inject $\left.{ }^{\circ}\right)$ patients. Recovery or improvement of AqCA was not apparent immediately after surgery. In some cases it took 4 weeks for an aqueous stream to become established following periods of fluctuation [90]. This may indicate a gradual process of SC or collector channel reperfusion, or realignment of homeostatic mechanisms. Unpredictable variations of IOP within the initial weeks after TBS likely occur due to these adjustments [90]. Variable longer-term IOP results seen with all forms of TBS and trabectome [93] prove the significance of post-trabecular outflow resistance and support the initial work of Grant $[18,20]$.

The episcleral venous system is a complex and heavily innervated venous web with multiple arterio-venous anastomoses (AVAs), characteristics that favour its potential involvement in IOP regulation [102]. There is some evidence that the EVS may modulate outflow resistance in mice. Smooth muscle was identified within vein walls of the distal AO tract using tissue labelling and two-photon microscopy [4]. This may indicate that veins within the episcleral system are built to respond to dynamic pressure changes in a similar way to blood pressure control.
None of the aforementioned theories of $\mathrm{AO}$ regulation consider the influence of episcleral venous pressure (EVP). The resistance generated by EVP opposes aqueous outflow and acts as a natural mechanism to prevent hypotony. Pathologically elevated EVP is well known to raise IOP [103]. EVP is challenging to measure accurately, especially non-invasively and in physiological conditions [103, 104]. This limits the study of EVP in the context of IOP regulation. However, the interaction between blood and aqueous within the EVS is evidence of a potential role for EVP in IOP control. Now, with HVI, visualisation of this dynamic relationship is possible $[87,90]$.

\section{Future directions}

The goal of AO imaging is to create an understanding of IOP control, which may enable targeted and personalised IOP-lowering treatments. We need to understand $\mathrm{AO}$ regulation to interpret inconsistent responses to current IOPlowering interventions; knowledge that may guide future treatment decisions. Vital information may be uncovered by observing physiological $\mathrm{AO}$ in an outpatient setting. Anterior segment OCT-A is an evolving technology, but aqueous cannot be resolved without the use of a contrast agent. Early work on ex vivo porcine eyes demonstrated the potential use of contrast in conjunction with anterior segment OCT-A [31, 43]. However, it may be our ability to visualise the response of AO to surgical and pharmacological interventions that allows us to unlock critical elements of IOP control. Real-time imaging techniques such as PhSOCT, aqueous angiography and HVI provide unique opportunities to capture such moments. Correlating imaging findings taken from the same patients may provide further clarity. None of these systems are currently available commercially and aqueous angiography is an invasive procedure. Technologies will evolve over time to improve efficiency and accessibility of AO imaging.

HVI is the most recent addition to the AO imaging techniques, and the first platform to deliver non-invasive imaging in the clinic setting under physiological conditions. This generates unique opportunities to quantify and compare AO within the EVS in many clinical scenarios. The immediate effects following selective laser trabeculoplasty [87] and short-term follow-up after TBS with iStent Inject [90] have been documented with HVI. Other interventions can be examined in a similar way. However, the identification of patients with glaucoma or staging the disease, is far more challenging. A normative database is required to achieve this goal and move towards personalised diagnosis and treatment. Quantification of AO needs to be refined, or even automated, before the technique can be used more widely. Nevertheless, HVI has the potential to uncover important discoveries that contribute to our understanding of IOP regulation. 
The AO system shares similarities with blood and lymphatic systems, both of which are regulated [6]. IOP regulation is most probably controlled by structures within the AO system [6]; therefore, imaging studies have concentrated on this region. Significant evidence has been amassed to support this theory including: segmental aqueous flow through TM, movement of TM in synchrony with ocular pulsation, the variable presence of pores along SC inner wall, valve-like structures permitting aqueous to enter and leave SC, AO pulsatility within episcleral veins, regional fluctuations of aqueous entering the EVS, the presence of AVAs within the EVS, and the variable reestablishment of distal AO following TBS. Despite this evidence there is no clarity regarding the influence of EVP and how the system functions in its entirety.

The contribution of aqueous production to IOP homeostasis also warrants consideration and may actively participate in the control of IOP. Theoretical feedback mechanisms between structures within the outflow system and the ciliary epithelium may exist to coordinate IOP regulation. Direct visualisation of a connection or communication between inflow and outflow systems is unlikely, but corroborating evidence may be uncovered, just as it has been within the AO system. Mechanosensing receptors may occur within sites of AO resistance; TM [6, 11], SC, or within episcleral vessel walls. Only recently has it become possible to image aqueous outflow within the EVS, making it possible at last to demonstrate feedback control of aqueous homeostasis.

\section{Conclusion}

Imaging is routinely relied upon to support medical diagnoses, determine treatment decisions and prognoses, and monitor progress. This stage has not yet been reached in glaucoma care largely due to an incomplete understanding of IOP dysregulation. Once that knowledge is attained then we predict that $\mathrm{AO}$ imaging will be used to improve patient outcomes in clinical glaucoma care. A body of knowledge has been built over a number of generations, driven by AO imaging and using a variety of imaging techniques. Each study acts as a building block for the next. Concurrent use of structural and functional imaging is likely to provide useful additional information. Non-invasive imaging techniques that assess structure and/or function of the $\mathrm{AO}$ pathways now exist (anterior segment OCT-A, PhS-OCT and HVI) and they can be performed under physiological conditions. Their commercial accessibility will promote further study of the distal AO pathway. We are in the unique position of using AO imaging to discover possible mechanisms for IOP homeostasis. Many challenges remain, but the ongoing development of novel or adapted imaging technologies will continue to assist the search for these mechanisms; information that may lead to the explanation of IOP homeostasis and elucidate the pathophysiology of primary open-angle glaucoma.

We have provided a detailed account of aqueous outflow imaging techniques and how they have contributed to our current understanding of aqueous outflow regulation. Noninvasive, physiological aqueous outflow analysis is now possible, and unlocks a new frontier in the study of IOP dysregulation in open-angle glaucoma.

Acknowledgements A core support grant from the Wellcome Trust and MRC to the Wellcome Trust-Medical Research Council Cambridge Stem Cell Institute. Haemoglobin Video Imaging facilities funded by Sydney Eye Hospital Foundation, Carl Zeiss Meditec, and Glaukos Corporation.

\section{Compliance with ethical standards}

Conflict of interest The authors declare that they have no conflict of interest.

Publisher's note Springer Nature remains neutral with regard to jurisdictional claims in published maps and institutional affiliations.

\section{References}

1. Ascher KW. The Aqueous Veins: I. Physiologic importance of the visible elimination of intraocular fluid. Am J Ophthalmol. 1942;25:1174-209.

2. Johnstone MA. The aqueous outflow system as a mechanical pump: evidence from examination of tissue and aqueous movement in human and non-human primates. J Glaucoma. 2004;13:421-38.

3. Johnstone MA, Martin E, Jamil A. Pulsatile flow into the aqueous veins: Manifestations in normal and glaucomatous eyes. Exp Eye Res. 2011;92:318-27.

4. Gonzalez JM Jr, Ko MK, Hong Y-K, Weigert R, Tan JCH. Deep tissue analysis of distal aqueous drainage structures and contractile features. Sci Rep. 2017;7:1701.

5. Johnstone MA. Intraocular pressure regulation: findings of pulsedependent trabecular meshwork motion lead to unifying concepts of intraocular pressure homeostasis. J Ocul Pharmacol Therapeutics. 2014;30:88-93.

6. Carreon T, van der Merwe E, Fellman R, Johnstone M, Bhattacharya SK. Aqueous outflow-a continuum from trabecular meshwork to episcleral veins. Prog Retin Eye Res. 2017;57:108-33.

7. Bill A, Phillips CI. Uveoscleral drainage of aqueous humor in human eyes. Exp Eye Res. 1971;12:275-81.

8. Huang AS, Francis BA, Weinreb RN. Structural and functional imaging of aqueous humour outflow: a review. Clin Experimen Ophthalmol. 2018;46:158-68.

9. Fautsch MP, Johnson DH. Second ARVO/Pfizer Research Institute Working Group. Aqueous humor outflow: what do we know? Where will it lead us?. Invest Ophthalmol Vis Sci. 2006;47:4181-7.

10. Ashton N. Anatomical study of Schlemm's canal and aqueous veins by means of neoprene casts. Part I Brit J Ophth. 1951;35:291. 
11. Goel M, Picciani RG, Lee RK, Bhattacharya SK. Aqueous humor dynamics: a review. Open Ophthalmol J. 2010;4:52-59.

12. Ujiie K, Bill A. The drainage routes for aqueous humor in monkeys as revealed by scanning electron microscopy of corrosion casts. Scan Electron Microsc. 1984; Pt2:849-56.

13. Smit BA, Johnstone MA. Effects of viscoelastic injection into Schlemm's canal in primate and human eyes: potential relevance to viscocanalostomy. Ophthalmology. 2002;109:786-92.

14. Van Buskirk EM. Anatomic correlates of changing aqueous outflow facility in excised human eyes. Invest Ophthalmol Vis Sci. 1982;22:625-32.

15. Overby D, Gong H, Qiu G, Freddo TF, Johnson M. The mechanism of increasing outflow facility during washout in the bovine eye. Invest Ophthalmol Vis Sci. 2002;43:3455-64.

16. Bentley MD, Hann CR, Fautsch MP. Anatomical variation of human collector channel orifices. Invest Ophthalmol Vis Sci. 2016;57:1153-9.

17. Tamm ER. The trabecular meshwork outflow pathways: structural and functional aspects. Exp Eye Res. 2009;88:648-55.

18. Grant WM. Experimental aqueous perfusion in enucleated human eyes. Arch Ophthalmol. 1963;69:783-801.

19. Maepea O, Bill A. Pressures in the juxtacanalicular tissue and Schlemm's canal in monkeys. Exp Eye Res. 1992;54:879-83.

20. Grant WM. Further studies on facility of flow through the trabecular meshwork. Arch Ophthal. 1958;60:523-33.

21. Smit BA, Johnstone MA. Effects of viscocanalostomy on the histology of Schlemm's canal in primate eyes. Invest Ophthalmol Vis Sci. 2000;41:S578.

22. Watson AM, Rose AH, Gibson GA, Gardner CL, Sun C, Reed DS, et al. Ribbon scanning confocal for high-speed highresolution volume imaging of brain. PLoS One. 2017;12: e0180486.

23. Loewen RT, Waxman S, Wang C, Atta S, Chen S, Watkins SC, et al. 3D-Reconstruction of the human conventional outflow system by ribbon scanning confocal microscopy. PLOS One. 2020;15:e0232833. https://doi.org/10.1371/journal.pone.0232833.

24. Francis AW, Kagemann L, Wollstein G, Ishikawa H, Folz S, Overby DR. Morphometric analysis of aqueous humor outflow structures with spectral-domain optical coherence tomography. Invest Ophthalmol Vis Sci. 2012;53:5198-207.

25. Waxman S, Loewen RT, Dang Y, Watkins SC, Watson AM, Loewen NA. High-resolution, three-dimensional reconstruction of the outflow tract demonstrates segmental differences in cleared eyes. Invest Ophthalmol Vis Sci. 2018;59:2371-80.

26. Zhang J, Ren L, Xi Mei, Xu Q, Zheng W, Liu Z. Microstructure visualization of conventional outflow pathway and finite element modeling analysis of trabecular meshwork. BioMed Eng OnLine. 2016;15 Suppl 2:162.

27. Li D, Zheng W, Qu JY. Two-photon autofluorescence microscopy of multicolor excitation. Opt Lett. 2009;34:202-4.

28. Hann CR, Bentley MD, Vercnocke A, Ritman EL, Fautsch MP. Imaging the aqueous humor outflow pathway in human eyes by three-dimensional micro-computed tomography (3D micro-CT). Exp Eye Res. 2011;92:104-11.

29. Jorgensen SM, Demirkaya O, Ritman EL. Three-dimensional imaging of vasculature and parenchyma in intact rodent organs with X-ray micro-CT. Am J Physiol. 1998;275:H1103-H1114.

30. Uji A, Muraoka Y, Yoshimura N. In vivo identification of the posttrabecular aqueous outflow pathway using swept-source optical coherence tomography. Invest Ophthalmol Vis Sci. 2016;57:4162-9.

31. Gottschalk HM, Wecker T, Khattab MH, Fischer CV, Callizo J, Rehfeldt F, et al. Lipid emulsion-based OCT angiography for ex vivo imaging of the aqueous outflow tract. Invest Ophthalmol Vis Sci. 2019;60:397-406.
32. Kagemann L, Wollstein G, Ishikawa H, Bilonick RA, Brennen PM, Folio LS, et al. Identification and assessment of Schlemm's canal by spectral-domain optical coherence tomography. Invest Ophthalmol Vis Sci. 2010;51:4054-9.

33. Kagemann L, Wollstein G, Ishikawa H, Sigal IA, Folio LS, Xu J, et al. 3D visualization of aqueous humor outflow structures insitu in humans. Exp Eye Res. 2011;93:308-15.

34. Ren J, Gille HK, Wu J, Yang C. Ex vivo optical coherence tomography imaging of collector channels with a scanning endoscopic probe. Invest Ophthalmol Vis Sci. 2011;52: 3921-5.

35. Li P, Reif R, Zhi Z, Martin E, Shen TT, Johnstone MA, et al. Phase-sensitive optical coherence tomography characterization of pulse-induced trabecular meshwork displacement in ex vivo nonhuman primate eyes. J Biomed Opt. 2012;17:076026.

36. Asrani S, Sarunic M, Santiago C, Izatt J. Detailed visualization of the anterior segment using fourier- domain optical coherence tomography. Arch Ophthalmol. 2008;126:765-71.

37. Sarunic MV, Asrani S, Izatt JA. Imaging the ocular anterior segment with real-time, full-range Fourier-domain optical coherence tomography. Arch Ophthalmol. 2008;126:537-42.

38. Akagi T, Uji A, Huang AS, Weinreb RN, Yamada T, Miyata M, et al. Conjunctival and intrascleral vasculatures assessed using anterior segment optic coherence tomography angiography in normal eyes. Am J Ophthalmol. 2018;196:1-9.

39. Shi G, Wang F, Li X, Lu J, Ding Z, Sun X, et al. Morphometric measurement of Schlemm's canal in normal human eye using anterior segment swept source optical coherence tomography. J Biomed Opt. 2012;17:016016.

40. Allingham RR, deKater AW, Ethier CR. Schlemm's canal and primary open angle glaucoma: correlation between Schlemm's canal dimensions and outflow facility. Exp Eye Res. 1996;62:101-9.

41. Chen Z, Sun J, Li M, Liu S, Chen L, Jing S, et al. Effect of age on the morphologies of the human Schlemm's canal and trabecular meshwork measured with swept-source optical coherence tomography. Eye. 2018;32:1621-8.

42. Yoshikawa M, Akagi T, Uji A, Nakanishi H, Kameda T, Suda K, et al. Pilot study assessing the structural changes in posttrabecular aqueous humor outflow pathway after trabecular meshwork surgery using swept-source optical coherence tomography. PLoS ONE. 2018;13:e0199739.

43. Wang B, Kagemann L, Schuman JS, Ishikawa H, Bilonick RA, Ling Y, et al. Gold nanorods as a contrast agent for Doppler optical coherence tomography. PLoS One. 2014;9:e90690.

44. Gao SS, Jia Y, Zhang M, Su JP, Liu G, Hwang TS, et al. Optical coherence tomography angiography. Invest Opthalmol Vis Sci. 2016;57:OCT27-OCT36.

45. Chen J, Huang H, Zhang S, Chen X, Sun X. Expansion of Schlemm's canal by travoprost in healthy subjects determined by fourier-domain optical coherence tomography. Invest Ophthalmol Vis Sci. 2013;54:1127-34.

46. Grieshaber MC, Pienaar A, Olivier J, Stegmann R. Clinical evaluation of the aqueous outflow system in primary open-angle glaucoma for canaloplasty. Invest Ophthalmol Vis Sci. 2010;51:1498-504.

47. Rosenquist R, Epstein D, Melamed S, Johnson M, Grant WM. Outflow resistance of enucleated human eyes at two different perfusion pressures and different extents of trabeculotomy. Curr Eye Res. 1989;8:1233-40.

48. Maurice DM. A new objective fluorophotometer. Exp Eye Res. 1963;2:33-38.

49. Langham M, Wybar KC. Fluorophotometric apparatus for the objective determination of fluorescence in the anterior chamber of the living eye. Br J Ophthalmol. 1954;38:52-57. 
50. Jones R, Maurice D. New methods of measuring the rate of aqueous flow in man with fluorescein. Exp Eye Res. 1966;5:208-20.

51. Nagataki S. Aqueous humor dynamics of human eyes as studied using fluorescein. Jpn J Ophthalmol. 1975;19:235.

52. Brubaker RF. Flow of aqueous humor in humans [The Friedenwald Lecture]. Invest Ophthalmol Vis Sci. 1991;32:3145-66.

53. Maus TL, Brubaker RF. Measurement of aqueous humor flow by fluorophotometry in the presence of a dilated pupil. Invest Ophthalmol Vis Sci. 1999;40:542-6.

54. Hann CR, Bahler CK, Johnson DH. Cationic ferritin and segmental flow through the trabecular meshwork. Invest Ophthalmol Vis Sci. 2005;46:1-7.

55. Sabanay I, Gabelt BT, Tian B, Kaufman PL, Geiger B. H-7 effects on the structure and fluid conductance of monkey trabecular meshwork. Arch Ophthalmol. 2000;118:955-62.

56. Ren R, Li G, Le TD, Kopczynski C, Stamer WD, Gong H. Netarsudil increases outflow facility in human eyes through multiple mechanisms. Invest Ophthalmol Vis Sci. 2016;57:6197-209.

57. Battista SA, Lu Z, Hofmann S, Freddo T, Overby DR, Gong H. Reduction of the available area for aqueous humor outflow and increase in meshwork herniations into collector channels following acute IOP elevation in bovine eyes. Invest Ophthalmol Vis Sci. 2008;49:5346-52.

58. Braakman ST, Read AT, Chan DW-H, Ethier CR, Overby DR. Colocalization of outflow segmentation and pores along the inner wall of Schlemm's canal. Exp Eye Res. 2015;130:87-96.

59. Keller KE, Bradley JM, Vranka JA, Acott TS. Segmental versican expression in the trabecular meshwork and involvement in outflow facility. Invest Ophthalmol Vis Sci. 2011;52:5049-57.

60. Karg SJ, Garron LK, Feeney ML, McEwen WK. Perfusion of human eyes with latex microspheres. Arch Ophthalmol. 1959;61:68-71.

61. Hann CR, Fautsch MP. Preferential fluid flow in the human trabecular meshwork near collector channels. Invest Ophthalmol Vis Sci. 2009;50:1692-7.

62. De Kater AW, Melamed S, Epstein DL. Patterns of aqueous humor outflow in glaucomatous and non-glaucomatous human eyes. A tracer study using cationized ferritin. Arch Ophthalmol. 1989;107:572-6.

63. Loewen RT, Brown EN, Roy P, Schuman JS, Sigal IA, Loewen NA. Regionally discrete aqueous humor outflow quantification using fluorescein canalograms. PLoS One. 2016;11:e0151754. https://doi.org/10.1371/journal.pone.0151754.

64. Loewen RT, Brown EN, Scott G, Parikh H, Schuman JS, Loewen NA. Quantification of focal outflow enhancement using differential canalograms. Invest Ophthalmol Vis Sci. 2016;57:2831-8.

65. Parikh HA, Loewen RT, Roy P, Schuman JS, Lathrop KL, Loewen NA. Differential canalograms detect outflow changes from trabecular micro-bypass stents and ab interno trabeculectomy. Sci Rep. 2016;6:34705.

66. Mendrinos E, Mermoud A, Shaarawy T. Nonpenetrating glaucoma surgery. Surv Ophthalmol. 2008;53:592-630.

67. Grieshaber MC. Ab externo Schlemm's canal surgery: viscocanalostomy and canaloplasty. Dev Ophthalmol. 2012;50:109-24.

68. Zeppa L, Ambrose L, Guerra G, Fortunato M, Costagliola G. In vivo near-infrared fluorescence imaging of aqueous humor outflow structures. J Ophthalmol. 2016;2016:8706564. https://doi. org/10.1155/2016/8706564.

69. Fellman RL, Grover DS. Episcleral venous fluid wave: intraoperative evidence of patency of the conventional outflow system. J Glaucoma. 2014;23:347-50.

70. Fellman RL, Feuer WJ, Grover DS. Episcleral venous fluid wave correlates with trabectome outcomes: intraoperative evaluation of the trabecular outflow pathway. Ophthalmology. 2015;122:2385-92.

71. Fellman RL, Grover DS. Episcleral Venous fluid wave in the living human eye adjacent to microinvasive glaucoma surgery (MIGS) supports laboratory research: outflow is limited circumferentially, conserved distally, and favored inferonasally. J Glaucoma. 2019;28:139-45.

72. Laroche D, Nortey A, Ng C. A novel use of trypan blue during canalicular glaucoma surgery to identify aqueous outflow to episcleral and intrascleral veins. J Glaucoma. 2018;27: e158-e161.

73. Saraswathy S, Tan JCH, Yu F, Francis BA, Hinton DR, Weinreb $\mathrm{RN}$, et al. Aqueous angiography: real-time and physiologic aqueous humor outflow imaging. PLoS One. 2016;11:e0147176.

74. Huang AS, Saraswathy S, Dastiridou A, Begian A, Legaspi H, Mohindroo $\mathrm{C}$, et al. Aqueous angiography with fluorescein and indocyanine green in bovine eyes. Transl Vis Sci Technol. 2016;5:5.

75. Huang AS, Saraswathy S, Dastiridou A, Begian A, Mohindroo $\mathrm{C}$, Tan $\mathrm{JCH}$, et al. Aqueous angiography-mediated guidance of trabecular bypass improves angiographic outflow in human enucleated eyes. Invest Ophthalmol Vis Sci. 2016;57:4558-65.

76. Huang AS, Li M, Yang D, Wang H, Wang N, Weinreb RN. Aqueous angiography in living nonhuman primates shows segmental, pulsatile, and dynamic angiographic aqueous humor outflow. Ophthalmology. 2017;124:793-803.

77. Huang AS, Camp A, BY X, Penteado RC, Weinreb RN. Aqueous angiography: aqueous humor outflow imaging in live human subjects. Ophthalmology. 2017;124:1249-51.

78. Huang A, Penteado RC, Papoyan V, Voskanyan L, Weinreb RN. Aqueous angiographic outflow improvement after trabecular microbypass in glaucoma patients. Ophthalmol Glaucoma. 2019;2:11-21.

79. Huang AS, Penteado RC, Saha SK, Do JL, Ngai P, Hu Z, et al. Fluorescein aqueous angiography in live normal human eyes. $\mathrm{J}$ Glaucoma 2018;27:957-64.

80. Ascher KW. Glaucoma and the aqueous veins. Am J Ophthalmol. 1942;25:1309-15.

81. Johnstone M, Stegmann R, Martin E, Jamil A. Pulsatile circumferential aqueous flow into Schlemm's Canal (SC) is synchronous with the cardiac pulse. Invest Ophthalmol Vis Sci. 2009;50:4855.

82. Li P, Shen TT, Johnstone M, Wang RK. Pulsatile motion of the trabecular meshwork in healthy human subjects quantified by phase-sensitive optical coherence tomography. Biomed Opt Express. 2013;4:2051-65.

83. Wang RK, Ma Z, Kirkpatrick SJ. Tissue Doppler optical coherence elastography for real time strain rate and strain mapping of soft tissue. Appl Phys Lett. 2006;89:144103.

84. Wang RK, Kirkpatrick SJ, Hinds M. Phase-sensitive optical coherence elastography for mapping tissue microstrains in real time. Appl Phys Lett. 2007;90:164105.

85. Johnstone MA. The aqueous outflow system as a mechanical pump: Evidence from examination of tissue and aqueous movement in human and non-human primates. J Glaucoma. 2004;13:421-38.

86. Meyer PA. The circulation of the human limbus. Eye. 1989;3:121-7.

87. Khatib TZ, Meyer PA, Lusthaus JA, Manyakin I, Mushtaq Y, Martin KR. Haemoglobin Video Imaging provides novel in vivo high-resolution imaging and quantification of human aqueous outflow in glaucoma patients. Ophthalmol Glaucoma. 2019;2:327-35

88. Meyer PA. Re-orchestration of blood flow by micro-circulations. Eye. 2018;32:222-9. 
89. Meyer PA. Anterior segment vascular imaging. In: Easty DL, Sparrow JM (eds) Oxford textbook of ophthalmology. Oxford University Press: Oxford, UK; 1999. pp 160-74.

90. Lusthaus JA, Meyer PAR, Khatib TZ, Martin KR. The effects of trabecular bypass surgery on conventional aqueous outflow, visualized by Hemoglobin Video Imaging. J Glaucoma. 2020;29:656-65.

91. Cha EDK, Xu J, Gong L, Gong H. Variations in active outflow along the trabecular outflow pathway. Exp Eye Res. 2016;146:354-60.

92. Johnson M. What controls aqueous humour outflow resistance? Exp Eye Res. 2006;82:545-57.

93. Overby DR, Stamer DW, Johnson M. The changing paradigm of outflow resistance generation: towards synergistic models of the JCT and inner wall endothelium. Exp Eye Res. 2009;88:656-70.

94. Johnson M, Shapiro A, Ethier CR, Kamm RD. Modulation of outflow resistance by the pores of the inner wall endothelium. Invest Ophthalmol Vis Sci. 1992;33:1670-5.

95. Ethier C, Chan D. Cationic ferritin changes outflow facility whereas anionic ferritin does not. Invest Ophthalmol Vis Sci. 2001;42:1795-802.

96. Stamer WD, Acott TS. Current understanding of conventional outflow dysfunction in glaucoma. Curr Opin Ophthalmol. 2012;23:135-43.

97. Wang K, Johnstone MA, Xin C, Song S, Padilla S, Vranka JA, et al. Estimating human trabecular meshwork stiffness by numerical modeling and advanced OCT imaging. Invest Ophthalmol Vis Sci. 2017;58:4809-17.

98. Xin C, Song S, Johnstone M, Wang N, Wang RK. Quantification of pulse-dependent trabecular meshwork motion in normal humans using phase-sensitive OCT. Invest Ophthalmol Vis Sci. 2018;59:3675-81.

99. Kagemann L, Wang B, Wollstein G, Ishikawa H, Nevins JE, Nadler Z, et al. IOP elevation reduces Schlemm's canal cross-sectional area. Invest Ophthalmol Vis Sci. 2014;55: 1805-9.

100. Johnstone MA, Grant WG. Pressure-dependent changes in structures of the aqueous outflow system of human and monkey eyes. Am J Ophthalmol. 1973;75:365-83.

101. Gulati V, Fan S, Hays CL, Samuelson TW, Ahmed IIK, Toris CB. A novel 8-mm Schlemm's canal scaffold reduces outflow resistance in a human anterior segment perfusion model. Invest Ophthalmol Vis Sci. 2013;54:1698-704.

102. Selbach JM, Rohen JW, Steuhl KP, Lutjen-Drecoll E. Angioarchitecture and innervation of the primate anterior episclera. Curr Eye Res. 2005;30:337-44.

103. Sit AJ, McLaren JW. Measurement of episcleral venous pressure. Exp Eye Res. 2011;93:291-8.

104. Lee SS, Robinson MR, Weinreb RN. Episcleral venous pressure and the ocular hypotensive effects of topical and intracameral prostaglandin analogs. J Glaucoma. 2019;28:846-57. 